\title{
Wavelets, Sobolev Multipliers, and Application to Schrödinger Type Operators with Nonsmooth Potentials
}

\author{
Pengtao Li, ${ }^{1}$ Qixiang Yang, ${ }^{2}$ and Yueping $\mathrm{Zhu}^{3}$ \\ ${ }^{1}$ Department of Mathematics, Shantou University, Shantou, Guangdong 515063, China \\ ${ }^{2}$ School of Mathematics and Statics, Wuhan University, Wuhan 430072, China \\ ${ }^{3}$ School of Sciences, Nantong University, Nantong 226007, China
}

Correspondence should be addressed to Qixiang Yang; qxyang@whu.edu.cn

Received 31 July 2013; Revised 17 September 2013; Accepted 18 September 2013

Academic Editor: Natig M. Atakishiyev

Copyright (C) 2013 Pengtao Li et al. This is an open access article distributed under the Creative Commons Attribution License, which permits unrestricted use, distribution, and reproduction in any medium, provided the original work is properly cited.

We employ Meyer wavelets to characterize multiplier space $X_{r, p}^{t}\left(\mathbb{R}^{n}\right)$ without using capacity. Further, we introduce logarithmic Morrey spaces $M_{r, p}^{t, \tau}\left(\mathbb{R}^{n}\right)$ to establish the inclusion relation between Morrey spaces and multiplier spaces. By fractal skills, we construct a counterexample to show that the scope of the index $\tau$ of $M_{r, p}^{t, \tau}\left(\mathbb{R}^{n}\right)$ is sharp. As an application, we consider a Schrödinger type operator with potentials in $M_{r, p}^{t, \tau}\left(\mathbb{R}^{n}\right)$.

\section{Introduction}

As useful tools, multipliers on the spaces of differential functions are applied to the study of various problems in harmonic analysis and differential equations. A function $g$ is called a multiplier from a Sobolev space $H^{t+r, p}\left(\mathbb{R}^{n}\right)$ to another Sobolev space $H^{t, p}\left(\mathbb{R}^{n}\right)$ if for every function $f \in H^{t+r, p}\left(\mathbb{R}^{n}\right)$, the product $f g \in H^{t, p}\left(\mathbb{R}^{n}\right)$. We denote by $X_{r, p}^{t}\left(\mathbb{R}^{n}\right)$ the class of all such functions $g$. In the famous book [1], Maz'ya and Shaposhnikova gave many characterizations of different kinds of multiplier spaces. For different indices $r, t$, and $p$, the multiplier spaces can adapt to the needs of different problems. Further, for different indices $r, t$, and $p$, they need different skills to deal with different multiplier spaces. In this paper, we consider multiplier spaces $X_{r, p}^{t}\left(\mathbb{R}^{n}\right)$ defined as follows.

Definition 1 (see [1]). Given $t \geq 0, r>0$ and $1<p<n /(r+t)$, the multiplier space $X_{r, p}^{t}\left(\mathbb{R}^{n}\right)$ is defined as the set of all the functions $f(x)$ such that

$$
\|f\|_{X_{r, p}^{t}\left(\mathbb{R}^{n}\right)}:=\sup _{\|g\|_{H^{t+r, p}\left(\mathbb{R}^{n}\right)} \leq 1}\|f g\|_{H^{t, p}\left(\mathbb{R}^{n}\right)}<\infty .
$$

For a compact set $e \subset \mathbb{R}^{n}$, the capacity $\operatorname{cap}\left(e, H^{t, p}\right)$ is defined by

$$
\operatorname{cap}\left(e, H^{t, p}\right)=\inf \left\{\|u\|_{H^{t, p}\left(\mathbb{R}^{n}\right)}^{p}: u \in \mathcal{S}, u \geq 1 \text { on } e\right\},
$$

where $\mathcal{S}$ denotes the Schwartz class of rapidly decreasing smooth functions on $\mathbb{R}^{n}$.

Lemma 2 (see [1]). Given $r>0$ and $t \geq 0$.

(i) For $1<p<n /(r+t), f \in X_{r, p}^{t}\left(\mathbb{R}^{n}\right)$ if and only if

$$
\sup _{e \subset \mathbb{R}^{n}}\left(\frac{\left\|(-\Delta)^{t / 2} f\right\|_{L^{p}(e)}}{\left(\operatorname{cap}\left(e, H^{t+r, p}\right)\right)^{1 / p}}+\frac{\|f\|_{L^{p}(e)}}{\left(\operatorname{cap}\left(e, H^{r, p}\right)\right)^{1 / p}}\right)<\infty .
$$

(ii) For $1<p<n / r$ and any cube $Q$ with a length less than 1 , the capacity $\operatorname{cap}\left(Q, H^{r, p}\right)$ is less than $C|Q|^{1-p r / n}$.

Our motivation is based on the following consideration. For complicated compact sets, it is very difficult to compute the capacity. The main aim of this paper is to establish a relation between $X_{r, p}^{t}\left(\mathbb{R}^{n}\right)$ and the Morrey spaces $M_{r, p}^{t}\left(\mathbb{R}^{n}\right)$, $t \geq 0$, and $r>0$ by wavelets. See also Liang et al. [2,3], Triebel 
[4], and Yuan et al. [5] for further information on wavelet characterization of Morrey spaces. The special case $t=0$ has been studied by Yang [6] and Yang and Zhou [7]. We point out that the result for $t>0$ is not a simple generalization. For $t=0$, the Sobolev space $H^{0, p}\left(\mathbb{R}^{n}\right)$ becomes Lebesgue space $L^{p}\left(\mathbb{R}^{n}\right)$. It is well known that

$$
\|f\|_{L^{p}} \sim\|M(f)\|_{L^{p}}, \quad 1<p<\infty
$$

where $M$ is the Hardy-Littlewood maximal operator. Yang and Zhou [7] used this equivalence to characterize $X_{r, p}^{0}\left(\mathbb{R}^{n}\right)$. See also Yang [6]. However, (4) does not hold for $H^{t, p}\left(\mathbb{R}^{n}\right), t>0$.

For the case $t>0$, it is necessary to make some progress in technique. The difficulty is to deal with the impact of the maximal operator $M$ on the frequencies when we split a product of two functions. To overcome this difficulty, we introduce an almost local operator $T^{t}$. See Definition 12. Let $r>0, t \geq 0$ with $t+r<1<p<n /(r+t)$. In Theorem 21, we characterize $X_{r, p}^{t}\left(\mathbb{R}^{n}\right)$ by Meyer wavelets without using capacity. Also our method can be applied to study the relation between multiplier spaces and Morrey spaces.

Lemma 2 implies that $X_{r, p}^{t}\left(\mathbb{R}^{n}\right) \subset M_{r, p}^{t}\left(\mathbb{R}^{n}\right)$. For the converse imbedding, Fefferman [8] established the following relation:

$$
M_{r, q}^{t}\left(\mathbb{R}^{n}\right) \subset X_{r, p}^{t}\left(\mathbb{R}^{n}\right), \quad q>p>1 .
$$

Let $f \in M_{r, p}^{t}\left(\mathbb{R}^{n}\right)$ and $g \in H^{t+r, p}\left(\mathbb{R}^{n}\right)$. From the counterexample in Theorem 33, we can see that the product $f g$ may produce a logarithmic type blowup on the fractal sets with Hausdorff dimension $n-p(r+t)$. To eliminate this defect, we introduce a logarithmic type Morrey space $M_{r, p}^{t, \tau}\left(\mathbb{R}^{n}\right)$ and prove that, for $\tau>1 / p^{\prime}$,

$$
M_{r, p}^{t, \tau}\left(\mathbb{R}^{n}\right) \subset X_{r, p}^{t}\left(\mathbb{R}^{n}\right),
$$

where $r>0, t \geq 0$, and $1<p<n /(r+t)$. See Section 4.1. In (6), the scope of $\tau$ is $\left(1 / p^{\prime}, \infty\right)$, where $1 / p+1 / p^{\prime}=1$. In Section 4.2, our counterexample implies that, for $0<$ $\tau \leq 1 / p^{\prime}$, there exists some function $f \in M_{r, p}^{t, \tau}\left(\mathbb{R}^{n}\right)$, but $f \notin X_{r, p}^{t}\left(\mathbb{R}^{n}\right)$. See Section 4.2 for the details. Theorems 24 and 33 illustrate the difference between Morrey spaces and multiplier spaces.

Remark 3. For the case $t=0$, some similar counterexamples have been obtained. Lemarié-Rieusset [9] gave a counterexample to show that $X_{r, 2}^{0}\left(\mathbb{R}^{n}\right) \neq M_{r, 2}^{0}\left(\mathbb{R}^{n}\right)$, where $n-2 r$ is an integer. Recently, Lemarié-Rieusset [10] and Yang and Zhou [7] constructed some counterexamples for $t=0$ and $1<$ $p<n / r$. The counterexamples there are independent of the wavelet characterization of $X_{r, p}^{t}\left(\mathbb{R}^{n}\right)$. Our counterexample depends on the wavelet characterization of multiplier space, Theorem 24, and fractal skills. See Theorem 33.

As an application, we apply our results obtained in Section 4 to the Schrödinger operator $L=I-\Delta+V$, where $V$ is the potential function. Maz'ya and Verbitsky [11] considered the multipliers from $H^{1,2}\left(\mathbb{R}^{n}\right)$ to $H^{-1,2}\left(\mathbb{R}^{n}\right)$. For a Schrödinger operator $L=I-\Delta+V$, they got many sufficient and necessary conditions such that $V$ is a multiplier from $H^{1,2}\left(\mathbb{R}^{n}\right)$ to $H^{-1,2}\left(\mathbb{R}^{n}\right)$. For more information, we refer the reader to Jiang et al. [12], Lemarié-Rieusset [9], Maz'ya and Shaposhnikova [1], Maz'ya and Verbitsky [11, 13], Yang and Yang [14], Yang et al. [15-17], Yang and Zhou [7], and the references therein.

Given $r>0, t \geq 0,1<p<n /(r+t)$, and $\tau>1 / p^{\prime}$, we consider the following equation:

$$
\left(I+(-\Delta)^{r / 2}+V\right) f=g
$$

where $g \in H^{t, p}\left(\mathbb{R}^{n}\right)$ and $V \in M_{r, p}^{t, \tau}\left(\mathbb{R}^{n}\right)$. If $V$ is a function of Hölder class, one usual method to deal with (7) is the boundedness of Calderón-Zygmund operators. As a function in $M_{r, p}^{t, \tau}\left(\mathbb{R}^{n}\right), V$ may not be a $L^{\infty}$ function. In Section 5, by Theorem 32, we prove that if $V \in M_{r, p}^{t, \tau}\left(\mathbb{R}^{n}\right),(7)$ has an unique solution in the Sobolev space $H^{t+r, p}\left(\mathbb{R}^{n}\right)$.

The rest of this paper is organized as follows. In Section 2, we state some notations and known results which will be used throughout this paper. In Section 3, we give a wavelet characterization of $X_{r, p}^{t}\left(\mathbb{R}^{n}\right)$. In Section 4 , we introduce a class of logarithmic Morrey spaces $M_{r, p}^{t, \tau}\left(\mathbb{R}^{n}\right)$ such that $M_{r, p}^{t, \tau}\left(\mathbb{R}^{n}\right) \subset$ $X_{r, p}^{t}\left(\mathbb{R}^{n}\right)$. Further, we construct a counterexample to prove the sharpness of the scope of the index $\tau$. In the last section, we consider an application to PDE problem.

Notations. $U \approx V$ represents that there is a constant $c>0$ such that $c^{-1} V \leq U \leq c V$ whose right inequality is also written as $U \lesssim V$. Similarly, if $V \geq c U$, we denote $V \gtrsim U$.

\section{Some Preliminaries}

In this section, we state some notations, knowledge, and preliminary lemmas which will be used in the sequel. Firstly, we recall some background knowledge of wavelets and multiresolution analysis.

For any $j \in \mathbb{N}$ and $k=\left(k_{1}, k_{2}, \ldots, k_{n}\right) \in \mathbb{Z}^{n}$, let $Q_{j, k}=$ $\prod_{s=1}^{n}\left[2^{-j} k_{s}, 2^{-j}\left(k_{s}+1\right)\right]$ and denote by $\Omega$ the set of all dyadic cubes $Q_{j, k}$. For arbitrary set $Q$, we denote by $\widetilde{Q}$ the $2^{M+2}$ multiple of $Q$. Finally, let $\chi, \widetilde{\chi}$ be the characteristic functions of the unit cube $Q_{0}$ and $\widetilde{Q}_{0}$, respectively.

We will adopt real-valued tensor product wavelets to study the multiplier spaces in this paper. Let $\left\{V_{j}^{1}, j \in \mathbb{Z}\right\}$ be an orthogonal multiresolution in $L^{2}(\mathbb{R})$ with the scaling function $\Phi^{0}(x)$. Denote by $W_{j}^{1}$ the orthogonal complement space of $V_{j}^{1}$ in $V_{j+1}^{1}$; that is, $W_{j}^{1}=V_{j+1}^{1} \ominus V_{j}^{1}$. Let $\left\{\Phi^{1}(x-k), k \in\right.$ $\mathbb{Z}\}$ be an orthogonal basis in $W_{0}^{1}$. For $\varepsilon=\left(\varepsilon_{1}, \ldots, \varepsilon_{n}\right) \in\{0,1\}^{n}$, denote $\Phi^{\varepsilon}(x)=\prod_{i=1}^{n} \Phi^{\varepsilon_{i}}\left(x_{i}\right)$.

In the proof, we use only Meyer wavelets and regular Daubechies wavelets. We say a Daubechies wavelet is regular 
if it has sufficient vanishing moment until order $m$ and $\Phi^{\varepsilon}(x) \in C_{0}^{m}\left(\left[-2^{M}, 2^{M}\right]^{n}\right)$, where the regularity exponent $m$ is large enough and $M$ is determined by $m$; see $[18,19]$ for more details. For any $\varepsilon \in\{0,1\}^{n}, j \in \mathbb{N}$, and $k \in \mathbb{Z}^{n}$, we denote $\Phi_{j, k}^{\varepsilon}(x)=2^{j n / 2} \Phi^{\varepsilon}\left(2^{j} x-k\right)$. For $j \in \mathbb{Z}$ and $k \in \mathbb{Z}^{n}$, let $\chi\left(2^{j} x-k\right)$ and $\tilde{\chi}\left(2^{j} x-k\right)$ be the characteristic functions on $Q_{j, k}$ and $\widetilde{Q}_{j, k}$, respectively. For simplicity, we denote by $\chi_{j, k}$ and $\tilde{\chi}_{j, k}$ for short.

In addition we define

$\Lambda_{n}=\left\{(\varepsilon, j, k): \varepsilon \in\{0,1\}^{n}, j \in \mathbb{N}, k \in \mathbb{Z}^{n}, \varepsilon \neq 0\right.$, if $\left.j>0\right\}$.

For fixed tempered distribution $f$, if we use wavelets which are sufficiently regular, then we can define $f_{j, k}^{\varepsilon}=\left\langle f, \Phi_{j, k}^{\varepsilon}\right\rangle$. And the wavelet representation $f=\sum_{(\varepsilon, j, k) \in \Lambda_{n}} f_{j, k}^{\varepsilon} \Phi_{j, k}^{\varepsilon}$ holds in the sense of distribution.

Let $\left\{V_{j}, j \in \mathbb{Z}^{n}\right\}$ be the orthogonal multiresolution in $L^{2}\left(\mathbb{R}^{n}\right)$ with the scaling function $\Phi^{0}(x)$. Denote by $W_{j}$ the orthogonal complement space of $V_{j}$ in $V_{j+1}$; that is, $W_{j}=$ $V_{j+1} \ominus V_{j}$. Denote by $P_{j}$ and $Q_{j}$ the projection operators from $L^{2}\left(\mathbb{R}^{n}\right)$ to $V_{j}$ and $W_{j}$, respectively. Dobynski got a decomposition of the product of two functions $f$ and $g$, which is similar to Bony's paraproduct (see [20]). Denote

$$
\begin{array}{r}
\widetilde{\Lambda}_{n}=\left\{\left(\varepsilon, \varepsilon^{\prime}, j, k, k^{\prime}\right), \varepsilon, \varepsilon^{\prime} \in\{0,1\}^{n} \backslash\{0\},\right. \\
\left.j \geq 0, k, k^{\prime} \in \mathbb{Z}^{n},(\varepsilon, k) \neq\left(\varepsilon^{\prime}, k^{\prime}\right)\right\} .
\end{array}
$$

By the projection operators $P_{j}$ and $Q_{j}$, we divide the product $f g$ into the following terms:

$$
\begin{aligned}
f(x) g(x)= & P_{0}(f) P_{0}(g)+\sum_{j \geq 0} P_{j}(f) Q_{j}(g) \\
& +\sum_{j \geq 0} Q_{j}(f) P_{j}(g)+\sum_{\Lambda_{n}} f_{j, k}^{\varepsilon} g_{j, k^{\prime}}^{\varepsilon^{\prime}} \Phi_{j, k}^{\varepsilon}(x) \Phi_{j, k^{\prime}}^{\varepsilon^{\prime}}(x) \\
& +\sum_{\Lambda_{n}, \varepsilon \neq 0} f_{j, k}^{\varepsilon} g_{j, k}^{\varepsilon}\left(\Phi_{j, k}^{\varepsilon}(x)\right)^{2} .
\end{aligned}
$$

To facilitate our use, we make a modification to (10) and use special wavelets for different cases. Let $N$ be a positive integer. We decompose the product $f g$ as

$$
\begin{aligned}
f g= & \sum_{j=1}^{\infty}\left[P_{j+1}(f) P_{j+1}(g)-P_{j}(f) P_{j}(g)\right]+P_{0}(f) P_{0}(g) \\
= & \sum_{j=N}^{\infty}\left[Q_{j}(f) Q_{j}(g)+P_{j}(f) Q_{j}(g)+Q_{j}(f) P_{j}(g)\right] \\
& +P_{N}(f) P_{N}(g)
\end{aligned}
$$

and the term $\sum_{j=N}^{\infty} Q_{j}(f) P_{j}(g)$ can be decomposed as

$$
\begin{aligned}
& \sum_{j=N}^{\infty} Q_{j}(f) P_{j}(g) \\
& \quad=\sum_{j=N}^{\infty} Q_{j}(f) \sum_{t=1}^{N} Q_{j-t}(g)+\sum_{j=N}^{\infty} Q_{j}(f) P_{j-N}(g) \\
& =\sum_{j=0}^{\infty} \sum_{t=1}^{N} Q_{j+t}(f) Q_{j}(g)+\sum_{j=0}^{\infty} Q_{j+N}(f) P_{j}(g) .
\end{aligned}
$$

In 1970s, Triebel introduced Triebel-Lizorkin spaces $F_{p}^{r, q}\left(\mathbb{R}^{n}\right)([21])$. Many function spaces can be seen as the special cases for $F_{p}^{r, q}\left(\mathbb{R}^{n}\right)$. For example, $F_{1}^{r, 2}\left(\mathbb{R}^{n}\right)$ is the fractional Hardy space. For $1<p<\infty, F_{p}^{r, 2}\left(\mathbb{R}^{n}\right)$ are the Sobolev spaces $H^{r, p}\left(\mathbb{R}^{n}\right)$. For $p=\infty, F_{\infty}^{-r, 2}\left(\mathbb{R}^{n}\right)$ is the space $\mathrm{BMO}^{r}\left(\mathbb{R}^{n}\right)$ defined as

$$
\mathrm{BMO}^{r}\left(\mathbb{R}^{n}\right):=(I-\Delta)^{-r / 2} \mathrm{BMO}_{\text {loc }}\left(\mathbb{R}^{n}\right),
$$

where $I$ and $\Delta$ denote the unit operator and the Laplace operator, respectively. Here $\mathrm{BMO}_{\mathrm{loc}}\left(\mathbb{R}^{n}\right)$ denotes the set of all measurable functions $f$ with

$$
\sup _{1 / 2 \leq l(Q) \leq 1}\left|f_{Q}\right|+\sup _{0<l(Q)<1 / 2}|Q|^{-1} \int_{Q}\left|f(x)-f_{Q}\right| d x<\infty,
$$

where $f_{\mathrm{Q}}$ denotes the mean value of $f$ on $Q$. See also Section 3.1 of [1].

For $1 \leq p<\infty$ and $r \in \mathbb{R}$, it is well known that $\left(F_{p}^{r, 2}\left(\mathbb{R}^{n}\right)\right)^{\prime}=F_{p^{\prime}}^{-r, 2}\left(\mathbb{R}^{n}\right)$. The following lemma gives a characterization of $F_{p}^{r, 2}\left(\mathbb{R}^{n}\right)$ via Meyer wavelets and regular Daubechies wavelets. For the proof, we refer the reader to Chapters 5 and 6 of Meyer [18]. See also Yuan et al. [22] and Yang [23].

Lemma 4. Given $|r|<m$, let $\left\{\Phi_{j, k}^{\varepsilon}\right\}$ be Meyer wavelets or $m$ regular Daubechies wavelets. Then,

(i) for $1 \leq p<\infty$,

$$
\begin{aligned}
g(x) & =\sum_{(\varepsilon, j, k) \in \Lambda_{n}} g_{j, k}^{\varepsilon} \Phi_{j, k}^{\varepsilon}(x) \in F_{p}^{r, 2}\left(\mathbb{R}^{n}\right) \\
& \Longleftrightarrow\left\|\left(\sum_{(\varepsilon, j, k) \in \Lambda_{n}} 2^{2 j(r+n / 2)}\left|g_{j, k}^{\varepsilon}\right|^{2} \chi_{j, k}(\cdot)\right)^{1 / 2}\right\|_{L^{p}}<\infty \\
& \Longleftrightarrow\left\|\left(\sum_{(\varepsilon, j, k) \in \Lambda_{n}} 2^{2 j(r+n / 2)}\left|g_{j, k}^{\varepsilon}\right|^{2} \tilde{\chi}_{j, k}(\cdot)\right)^{1 / 2}\right\|_{L^{p}}<\infty,
\end{aligned}
$$

(ii) $g(x)=\sum_{(\varepsilon, j, k) \in \Lambda_{n}} g_{j, k}^{\varepsilon} \Phi_{j, k}^{\varepsilon}(x) \in F_{\infty}^{r, 2}\left(\mathbb{R}^{n}\right)$ if and only if there exists $p \in(1, \infty)$ such that for $Q \in \Omega$,

$$
\left\|\left(\sum_{\varepsilon \in E_{n}, Q_{j, k} \subset Q} 2^{2 j(r+n / 2)}\left|g_{j, k}^{\varepsilon}\right|^{2} \chi_{j, k}(\cdot)\right)^{1 / 2}\right\|_{L^{p}} \leq C|Q|^{1 / p} .
$$


The wavelet characterizations of function spaces have been studied by many authors. In [18, Chapters 5 and 6], Meyer established wavelet characterizations for many function spaces, for example, Hardy space, BMO spaces, Besov spaces, and Bloch space. Yang et al. [24] used wavelets to characterize Lorentz type Triebel-Lizorkin spaces and Lorentz type Besov spaces. For the wavelet characterization of Besov type Morrey spaces and Triebel-Lizorkin type Morrey spaces, We refer to Yang and Yuan [25, 26], Yang [23], and Yuan et al. [22].

Morrey spaces were introduced by Morrey in 1938 and played an important role in the research of partial differential equations. Xiao [27] established a relation between the homogeneous Morrey space $\mathscr{L}^{2, n-2 \alpha}\left(\mathbb{R}^{n}\right)$ and $Q$-type space $Q_{\alpha}\left(\mathbb{R}^{n}\right)$ by heat semigroup and the fractional integrals. In the recent 20 years, $Q$-type spaces are studied extensively. See Essèn et al. [28], Dafni and Xiao [29, 30], Peng and Yang [31], $\mathrm{Wu}$ and Xie [32], Yang [23], and Yuan et al. [22].

Define $f_{t, Q}$ as

$$
f_{t, Q}=\frac{1}{|Q|} \int_{Q}(I-\Delta)^{t / 2} f(x) d x
$$

The Morrey spaces $M_{r, p}^{t}\left(\mathbb{R}^{n}\right)$ are defined as follows.

Definition 5. Given $1 \leq p<\infty$ and $r, t \geq 0$, the Morrey space $M_{r, p}^{t}\left(\mathbb{R}^{n}\right)$ is defined as the set of all measurable functions $f$ such that $\sup _{|Q|=1}\left|f_{t, Q}\right| \leq C$ and

$$
\int_{Q}\left|(I-\Delta)^{t / 2} f(x)-f_{t, \mathrm{Q}}\right|^{p} d x \leq C|Q|^{1-p(r+t) / n}
$$

where $Q$ is any cube in $\mathbb{R}^{n}$ with $|Q| \leq 1$.

Morrey spaces $M_{r, p}^{t}\left(\mathbb{R}^{n}\right)$ can be also characterized by wavelets. We state it as the following theorem and refer to Yuan et al. [22] for the proof.

Theorem 6. Given $t \in \mathbb{R}, 1<p<\infty$ and $0 \leq p(r+t)<n$,

$$
f(x)=\sum_{(\varepsilon, j, k) \in \Lambda_{n}} f_{j, k}^{\varepsilon} \Phi_{j, k}^{\varepsilon}(x) \in M_{r, p}^{t}\left(\mathbb{R}^{n}\right)
$$

if and only if for any $Q \in \Omega$ with $|Q| \leq 1$

$$
\int_{Q}\left(\sum_{\varepsilon \in E_{n}, Q_{j, k} \subset Q} 2^{j(n+2 t)}\left|f_{j, k}^{\varepsilon}\right|^{2} \chi_{j, k}(x)\right)^{p / 2} d x \leq C|Q|^{1-p(r+t) / n} .
$$

The following lemma can be obtained by Lemmas 2 and 4 immediately.

Lemma 7. Given $r>0, t \geq 0$ and $1<p<n /(r+t)$, then $X_{r, p}^{t}\left(\mathbb{R}^{n}\right) \subset M_{r, p}^{t}\left(\mathbb{R}^{n}\right)$.

Now we give two lemmas about $\mathrm{BMO}^{r}\left(\mathbb{R}^{n}\right)$.
Lemma 8. Given $r>0, t \geq 0$ and $1<p<n /(r+t)$, then $M_{r, p}^{t}\left(\mathbb{R}^{n}\right) \subset B M O^{r}\left(\mathbb{R}^{n}\right)$.

Proof. For any dyadic cube $Q$, we have

$$
\begin{aligned}
& \int\left(\sum_{\varepsilon \in E_{n}, Q_{j, k} \subset Q} 2^{j n-2 j r}\left|f_{j, k}^{\varepsilon}\right|^{2} \chi_{j, k}(x)\right)^{p / 2} d x \\
& \quad \leq|Q|^{p(r+t) / n} \int\left(\sum_{\varepsilon \in E_{n}, Q_{j, k} \subset Q} 2^{j(n+2 t)}\left|f_{j, k}^{\varepsilon}\right|^{2} \chi_{j, k}(x)\right)^{p / 2} d x \\
& \quad \leq|Q| .
\end{aligned}
$$

For any $\varepsilon \in\{0,1\}^{n}, j \in \mathbb{N}$ and $k \in \mathbb{Z}^{n}$, we denote $f_{j, k}^{\varepsilon}=$ $\left\langle f, \Phi_{j, k}^{\varepsilon}\right\rangle$. We can get the following result.

Lemma 9. Suppose that $r>0$ and $f \in B M O^{r}\left(\mathbb{R}^{n}\right)$. The wavelet coefficients of $f$ satisfy

$$
\left|f_{j, k}^{\varepsilon}\right| \lesssim 2^{(r-n / 2) j}, \quad \forall \varepsilon \in 1\{0,1\}^{n}, j \in \mathbb{N}, k \in \mathbb{Z}^{n} .
$$

Proof. Take $j \in \mathbb{N}$ and $k \in \mathbb{Z}^{n}$. We consider two cases $\varepsilon \in E_{n}$ and $\varepsilon=0$ separately.

(i) For $\varepsilon \in E_{n}$, by Lemma 4, we get

$$
\int\left(2^{j n-2 j r}\left|f_{j, k}^{\varepsilon}\right|^{2} \chi_{j, k}(x)\right)^{p / 2} d x \leqslant 2^{-j n}
$$

It is easy to see that $\left|f_{j, k}^{\varepsilon}\right| \lesssim 2^{j(r-n / 2)}$.

(ii) For $\varepsilon=0$,

$$
\begin{aligned}
f_{j, k}^{0} & =\left\langle\sum_{\left(\varepsilon^{\prime}, j^{\prime}, k^{\prime}\right) \in \Lambda_{n}} f_{j^{\prime}, k^{\prime}}^{\varepsilon^{\prime}} \Phi_{j^{\prime}, k^{\prime}}^{\varepsilon^{\prime}}, \Phi_{j, k}^{0}\right\rangle \\
& =\left\langle\sum_{\left(\varepsilon^{\prime}, j^{\prime}, k^{\prime}\right) \in \Lambda_{n}: j^{\prime}<j} f_{j^{\prime}, k^{\prime}}^{\varepsilon^{\prime}} \Phi_{j^{\prime}, k^{\prime}}^{\varepsilon^{\prime}}, \Phi_{j, k}^{0}\right\rangle .
\end{aligned}
$$

Because $\left|\sum_{\left(\varepsilon^{\prime}, j^{\prime}, k^{\prime}\right) \in \Lambda_{n}: j^{\prime}<j} f_{j^{\prime}, k^{\prime}}^{\varepsilon^{\prime}} \Phi_{j^{\prime}, k^{\prime}}^{\varepsilon^{\prime}}(x)\right| \lesssim 2^{r j}$, we have

$$
\left|f_{j, k}^{0}\right| \lesssim\left\langle 2^{r j},\left|\Phi_{j, k}^{0}(x)\right|\right\rangle \lesssim 2^{j(r-n / 2)}
$$

Let $\Psi^{1}$ and $\Psi^{2}$ be two functions such that

$$
\begin{gathered}
\Psi^{i} \in C_{0}^{\mu}\left(\left[-2^{M+1}, 2^{M+1}\right]^{n}\right), \quad i=1,2, \\
\int x^{\alpha} \Psi^{i}(x) d x=0, \quad \text { where }|\alpha| \leq \mu, \quad i=1,2 .
\end{gathered}
$$

Write $a_{j, k, j^{\prime}, k^{\prime}}=\left\langle\Psi_{j, k}^{1}, \Psi_{j^{\prime}, k^{\prime}}^{2}\right\rangle$. The following lemma can be found in Chapter 8 of Meyer [18] or Chapter 6 of Yang [23]. 
Lemma 10 (see [18, Chapter 8, Lemma 1]). Let $|\mu| \leq m$. For $|s|<\mu$, the coefficients $a_{j, k, j^{\prime}, k^{\prime}}$ satisfy the following condition:

$$
\left|a_{j, k, j^{\prime}, k^{\prime}}\right| \lesssim 2^{-\left|j-j^{\prime}\right|(n / 2+s)}\left(\frac{2^{-j}+2^{-j^{\prime}}}{2^{-j}+2^{-j^{\prime}}+\mid k 2^{-j}-k^{\prime} 2^{-j^{\prime} \mid}}\right)^{n+s} .
$$

By Lemma 4, the boundedness of Calderón-Zygmund operators on $H^{r, p}\left(\mathbb{R}^{n}\right)$ is equivalent to the following lemma. We refer the reader to $[18,23,33]$ for the proof.

Lemma 11. Suppose that $s>|r|$ and $g(x)=$ $\sum_{(\varepsilon, j, k) \in \Lambda_{n}} g_{j, k}^{\varepsilon} \Phi_{j, k}^{\varepsilon}(x) \quad \in \quad H^{r, p}\left(\mathbb{R}^{n}\right)$. Let $\widetilde{\mathcal{g}}_{j, k}^{\varepsilon}=$ $\sum_{(\varepsilon, j, k) \in \Lambda_{n}} a_{j, k, j^{\prime}, k^{\prime}}^{\varepsilon, \varepsilon^{\prime}} g_{j^{\prime}, k^{\prime}}^{\varepsilon}$. If the coefficients $a_{j, k, j^{\prime}, k^{\prime}}^{\varepsilon, \varepsilon^{\prime}}$ satisfy (27), then

$$
\begin{aligned}
& \int\left(\sum_{(\varepsilon, j, k) \in \Lambda_{n}} 2^{j(n+2 r)}\left|\tilde{g}_{j, k}^{\varepsilon}\right|^{2} \chi_{j, k}(x)\right)^{p / 2} d x \\
& \quad \lesssim \int\left(\sum_{(\varepsilon, j, k) \in \Lambda_{n}} 2^{j(n+2 r)}\left|g_{j, k}^{\varepsilon}\right|^{2} \chi_{j, k}(x)\right)^{p / 2} d x .
\end{aligned}
$$

We say that $T$ is a local operator if there exists some constant $C>1$ such that, for all $x \in \mathbb{R}^{n}$ and $r>0, T$ maps a distribution with the support $B(x, r)$ to another distribution supported on the ball $B(x, C r)$. If $t / 2$ is not a nonnegative integer, the operator $(I-\Delta)^{t / 2}$ is not a local operator. Now we use wavelets to construct some special fractional differential operators $T^{t}$, which are almost local operators and will be used in the proof of our main result.

Definition 12. For $t \geq 0$ and $h(x)=\sum_{(\varepsilon, j, k) \in \Lambda_{n}} h_{j, k}^{\varepsilon} \Phi_{j, k}^{\varepsilon}(x)$, we call that $T^{t}$ is an operator associated to the kernel $K^{t}(x, y)=$ $\sum_{(\varepsilon, j, k) \in \Lambda_{n}} 2^{-j t} \Phi_{j, k}^{\varepsilon}(x) \Phi_{j, k}^{\varepsilon}(y)$ if

$$
T^{t} h(x)=\sum_{(\varepsilon, j, k) \in \Lambda_{n}} 2^{-j t} h_{j, k}^{\varepsilon} \Phi_{j, k}^{\varepsilon}(x) .
$$

It is easy to prove that $T^{0}$ is the identity operator and $\left\|T^{t} h\right\|_{L^{p}}=\|h\|_{H^{-t, p}}$ for $1<p<\infty$. Furthermore, we have the following.

Lemma 13. Suppose $t \geq 0$. For any $Q_{j, k} \in \Omega$ and $x \in Q_{j, k}$,

$$
2^{j(n / 2-t)}\left|h_{j, k}^{0}\right| \leq C M T^{t} h(x)
$$

where $M$ is the Hardy-Littlewood maximal operator.

Proof. If $t=0$, the proof was given by Meyer [18]. Now we consider the case $t>0$. It is easy to verify that

$$
\left|T^{-t} \Phi^{0}(x)\right|=\left|\int K^{-t}(x, y) \Phi^{0}(y) d y\right| \leq C(1+|x|)^{-n-t} .
$$

By the fact that $t>0$, we have

$$
\begin{aligned}
2^{j(n / 2-t)} h_{j, k}^{0} & =2^{j(n / 2-t)}\left\langle T^{t} h, T^{-t} \Phi_{j, k}^{0}\right\rangle \\
& =2^{j n / 2}\left\langle T^{t} h,\left(T^{-t} \Phi^{0}\right)_{j, k}\right\rangle .
\end{aligned}
$$

Hence, we can get

$$
\begin{aligned}
& \left|2^{j(n / 2-t)} h_{j, k}^{0}\right| \\
& \quad=2^{j n / 2}\left|\left\langle T^{t} h,\left(T^{-t} \Phi^{0}\right)_{j, k}\right\rangle\right| \\
& \quad \leq 2^{j n / 2} \int\left|T^{t} h(x)\right| 2^{j n / 2} \frac{d x}{\left(1+\left|2^{j} x-k\right|\right)^{n+t}} \\
& \quad \leq 2^{j n}\left(\int_{\left|2^{j} x-k\right| \leq 1}\left|T^{t} h(x)\right| d x\right. \\
& \left.\quad+\sum_{l=1}^{\infty} \int_{2^{l-1} \leq 2^{j} x-k \mid \leq 2^{l}}\left|T^{t} h(x)\right| \frac{d x}{\left(1+\left|2^{j} x-k\right|\right)^{n+t}}\right) \\
& \quad \leq 2^{j n}\left(2^{-j n} M\left(T^{t} h\right)(x)+\sum_{l=1}^{\infty} 2^{-l t} M\left(T^{t} h\right)(x) 2^{-j n}\right) \\
& \quad \leq M\left(T^{t}\right) h(x) .
\end{aligned}
$$

This completes the proof of Lemma 13.

In the rest of this section, we give a decomposition of Sobolev spaces associated with combination atoms. For $|r|<$ $m$ and $g(x)=\sum_{(\varepsilon, j, k) \in \Lambda_{n}} g_{j, k}^{\varepsilon} \Phi_{j, k}^{\varepsilon}(x)$, denote

$$
S_{r} g(x)=\left(\sum_{(\varepsilon, j, k) \in \Lambda_{n}} 2^{j(2 r+n)}\left|g_{j, k}^{\varepsilon}\right|^{2} \chi_{j, k}(x)\right)^{1 / 2},
$$

and, for $r=0$, denote also $S g(x)=S_{0} g(x)$.

Definition 14. Let $r \in \mathbb{R}$ and $\lambda>0$. For arbitrary measurable set $E$ with finite measure, we say that $g(x)$ is a $(r, \lambda, E)$ combination atom if $\operatorname{supp}\left(S_{r} g\right) \subset E$ and $S_{r} g(x) \leq \lambda$. If $E$ is a dyadic cube, then we say $g(x)$ is a $(r, \lambda, E)$-atom.

In the next theorem, we give a combination atom decomposition of Sobolev spaces. This result is a generalization of that of Yang [34].

Theorem 15. If $1<p<\infty,|r|<m$ and $\|g\|_{H^{r, p}} \leq 1$, there exists a series of $\left(r, 2^{v}, E_{v}\right)$-combination atoms $g_{v}(x)$ such that $\sum_{v \in \mathbb{N}} 2^{p v}\left|E_{v}\right| \leq C$.

Proof. Denote

$$
\widetilde{S}_{r} g(x)=\left(\sum_{(\varepsilon, j, k) \in \Lambda_{n}} 2^{j(2 r+n)}\left|g_{j, k}^{\varepsilon}\right|^{2} \widetilde{\chi}_{j, k}(x)\right)^{1 / 2} .
$$


For $v \geq 1$, let $E_{v}=\left\{x: \widetilde{S}_{r} g(x)>2^{v}\right\}$. By wavelet characterization of Sobolev spaces, we have $\sum_{v \in \mathbb{N}} 2^{p v}\left|E_{v}\right| \lesssim 1$. Let $E_{v}=\bigcup_{l} Q^{v, l}$, where $Q^{v, l}$ are disjoint maximal dyadic cubes with $\left|Q^{v, l}\right| \leq 1$. Let $\mathfrak{\Im}_{v, l}$ be the set of dyadic cubes contained in $Q^{v, l}$ but not in $E_{v+1}, \mathfrak{F}_{v}=\bigcup_{l} \mathfrak{F}_{v, l}$ and $\mathfrak{F}_{0}=\Omega \backslash \bigcup_{v \geq 1} \mathfrak{F}_{v}$. Let $E_{0}=\left\{x \in Q, Q \in \mathfrak{F}_{0}\right\}$, and we can write also $E_{0}=\bigcup_{l} Q^{0, l}$, where $Q^{0, l}$ are disjoint maximal dyadic cubes in $\Omega$. The related set $\mathfrak{F}_{0, l}$ is defined as $\mathfrak{F}_{0, l}=\left\{Q \subset Q^{0, l}\right.$ and $\left.Q \in \mathfrak{F}_{0}\right\}$.

For any $v \geq 0$, we write

$$
\begin{gathered}
g_{v, l}(x)=\sum_{Q_{j, k} \in \mathscr{F}_{v, l}} g_{j, k}^{\varepsilon} \Phi_{j, k}^{\varepsilon}(x), \\
g_{v}(x)=\sum_{Q_{j, k} \in \widetilde{F}_{v}} g_{j, k}^{\varepsilon} \Phi_{j, k}^{\varepsilon}(x) .
\end{gathered}
$$

Then $g_{v}(x)$ is a desired combination atom. This completes the proof.

\section{Wavelet Characterization of the Multiplier Spaces}

In this section, we use Meyer wavelets to characterize $X_{r, p}^{t}\left(\mathbb{R}^{n}\right)$. Let $\Phi$ be a function in $C_{0}^{\infty}(B(0,1))$ satisfying $\Phi(x) \geq 0$ and $\int \Phi(x) d x=1$. For any $g \in H^{t, p}\left(\mathbb{R}^{n}\right)$, define

$$
g_{j, k}^{\Phi}=\left\langle g, 2^{j n} \Phi\left(2^{j} \cdot-k\right)\right\rangle .
$$

The function space $S_{r, p}^{\Phi, t}\left(\mathbb{R}^{n}\right)$ is defined as follows.

Definition 16. Given $r>0, t \geq 0$ and $r+t<1<p<n /(r+t)$, we say $f \in S_{r, p}^{\Phi, t}\left(\mathbb{R}^{n}\right)$ if $f(x)=\sum_{(\varepsilon, j, k) \in \Lambda_{n}} f_{j, k}^{\varepsilon} \Phi_{j, k}^{\varepsilon}(x)$ and

$$
\int\left(\sum_{(\varepsilon, j, k) \in \Lambda_{n}} 2^{j(n+2 t)}\left|g_{j, k}^{\Phi}\right|^{2}\left|f_{j, k}^{\varepsilon}\right|^{2} \chi_{j, k}(x)\right)^{p / 2} d x \leqslant\|g\|_{H^{r+t, p}},
$$

where $g \in H^{r+t, p}\left(\mathbb{R}^{n}\right)$ and $\|g\|_{H^{r+t, p}} \leq 1$.

Now we give a wavelet characterization of $X_{r, p}^{t}\left(\mathbb{R}^{n}\right)$. Let $\Phi^{0}$ and $\Phi^{\varepsilon}, \varepsilon \in E_{n}$, be the scaling function and wavelet functions, respectively. For $(\varepsilon, j, k),\left(\varepsilon^{\prime}, j^{\prime}, k^{\prime}\right),\left(\varepsilon^{\prime \prime}, j^{\prime}, k^{\prime}\right) \in \Lambda_{n}$ and $l \in \mathbb{Z}^{n}$, let

$$
\begin{gathered}
a_{j, k, l, j^{\prime}, k^{\prime}}^{\varepsilon, \varepsilon^{\prime}}=\left\langle\Phi_{j, k+l}^{0} \Phi_{j, k}^{\varepsilon}, \Phi_{j^{\prime}, k^{\prime}}^{\varepsilon^{\prime}}\right\rangle, \\
a_{j, k, 0, j^{\prime}, k^{\prime}}^{\varepsilon, \varepsilon^{\prime \prime}}=\left\langle\left(\Phi_{j, k}^{\varepsilon}\right)^{2}-2^{j n} \Phi\left(2^{j} \cdot-k\right), \Phi_{j^{\prime}, k^{\prime}}^{\varepsilon^{\prime \prime}}\right\rangle .
\end{gathered}
$$

Furthermore, for $0 \leq s \leq N, \varepsilon^{\prime} \in E_{n}, l \in \mathbb{Z}^{n}$, and $s+\left|\varepsilon-\varepsilon^{\prime}\right|+$ $|l| \neq 0$, let

$$
a_{j, k, l, j^{\prime}, k^{\prime}}^{\varepsilon, \varepsilon^{\prime}, \varepsilon^{\prime \prime}, s}=\left\langle\Phi_{j, k}^{\varepsilon^{\prime}} \Phi_{j+s, 2^{2} k+l}^{\varepsilon}, \Phi_{j^{\prime}, k^{\prime}}^{\varepsilon^{\prime \prime}}\right\rangle .
$$

By the same method of [18, Chapter 8 , Lemma 1], we could prove the following lemma.
Lemma 17. There exist sufficient big integers $N, N_{1}$, and $N_{2}$ such that $\min \left\{N, N_{1}, N_{2}\right\}>8 n+8 m$ and the following estimates hold.

(i) If $(\varepsilon, j, k),\left(\varepsilon^{\prime}, j^{\prime}, k^{\prime}\right) \in \Lambda_{n}, l \in \mathbb{Z}^{n}$, and $j \geq j^{\prime}$, then

$$
\left|a_{j, k, l, j^{\prime}, k^{\prime}}^{\varepsilon, \varepsilon^{\prime}}\right| \leqslant(1+|l|)^{-N_{1}} 2^{n j^{\prime} / 2+j^{\prime}-j}\left(1+\left|k^{\prime}-2^{j^{\prime}-j} k\right|\right)^{-N_{2}} .
$$

(ii) If $(\varepsilon, j, k),\left(\varepsilon^{\prime \prime}, j^{\prime}, k^{\prime}\right) \in \Lambda_{n}, 0 \leq s \leq N, \varepsilon^{\prime} \in E_{n}, l \in \mathbb{Z}^{n}$, and $j \geq j^{\prime}$, then

$$
\left|a_{j, k, l, j^{\prime}, k^{\prime}}^{\varepsilon, \varepsilon^{\prime}, \varepsilon^{\prime \prime}, s}\right| \lesssim(1+|l|)^{-N_{1}} 2^{n j^{\prime} / 2+j^{\prime}-j}\left(1+\left|k^{\prime}-2^{j^{\prime}-j} k\right|\right)^{-N_{2}} .
$$

(iii) If $(\varepsilon, j, k),\left(\varepsilon^{\prime}, j^{\prime}, k^{\prime}\right) \in \Lambda_{n}, l \in \mathbb{Z}^{n}$, and $j<j^{\prime}$, then

$$
\left|a_{j, k, l, j^{\prime}, k^{\prime}}^{\varepsilon, \varepsilon^{\prime}}\right| \leqslant(1+|l|)^{-N_{1}} 2^{-n j^{\prime} / 2+n j+N\left(j-j^{\prime}\right)}\left(1+\left|k-2^{j-j^{\prime}} k^{\prime}\right|\right)^{-N_{2}} .
$$

(iv) If $(\varepsilon, j, k),\left(\varepsilon^{\prime \prime}, j^{\prime}, k^{\prime}\right) \in \Lambda_{n}, 0 \leq s \leq N, \varepsilon^{\prime} \in E_{n}, l \in \mathbb{Z}^{n}$, and $j<j^{\prime}$, then

$\left|a_{j, k, l, j^{\prime}, k^{\prime}}^{\varepsilon, \varepsilon^{\prime}, \varepsilon^{\prime \prime}, s}\right| \leqslant(1+|l|)^{-N_{1}} 2^{-n j^{\prime} / 2+n j+N\left(j-j^{\prime}\right)}\left(1+\left|k-2^{j-j^{\prime}} k^{\prime}\right|\right)^{-N_{2}}$.

3.1. Decomposition of Products via Multilinear Analysis. At first, we give a wavelet decomposition of the product of $f h$. Let $\Phi^{0}$ and $\left\{\Phi^{\varepsilon}, \varepsilon \in E_{n}\right\}$ be the scaling function and wavelet functions of Meyer wavelets, respectively. There exists an integer $N \geq 3$ such that

$$
\int x^{\alpha} \Phi^{0}(x) \Phi^{\varepsilon}\left(2^{N} x-k\right) d x=0
$$$$
\text { for any } k \in \mathbb{Z}^{n}, \alpha \in \mathbb{N}^{n}, \varepsilon \in E_{n} \text {. }
$$

Denote

$$
\begin{gathered}
\Lambda_{\varepsilon, n}=\left\{\left(s, \varepsilon^{\prime}, l\right), 0 \leq s \leq N, \varepsilon^{\prime} \in E_{n}, l \in \mathbb{Z}^{n},|l| \leq 2^{(M+2+s) n},\right. \\
\left.\quad \text { and if } s=0,(0, \varepsilon, 0) \neq\left(0, \varepsilon^{\prime}, 0\right)\right\}, \\
\mathbb{Z}_{N}^{n}=\left\{l=\left(l_{1}, \ldots, l_{n}\right) \in \mathbb{Z}^{n}, 0 \leq l_{i} \leq 2^{N}-1, i=1, \ldots, n\right\} .
\end{gathered}
$$


For $\varepsilon \in E_{n}, l \in \mathbb{Z}^{n},|l| \leq 2^{(M+2) n}$, and $\left(\varepsilon^{\prime}, l\right) \in \Lambda_{\varepsilon, n}$, we denote

$$
\begin{aligned}
& T_{1, \varepsilon}(x)=\sum_{j \in \mathbb{N}, k \in \mathbb{Z}^{n}} \sum_{l \in \mathbb{Z}^{n}} f_{j, k+l}^{0} h_{j, k}^{\varepsilon} \Phi_{j, k+l}^{0}(x) \Phi_{j, k}^{\varepsilon}(x), \\
& T_{2,0, \varepsilon, \varepsilon}(x)= \sum_{j \in \mathbb{Z}, k \in \mathbb{Z}^{n}} \sum_{l \in \mathbb{Z}^{n}, l \neq 0} f_{j, k+l}^{\varepsilon} h_{j, k}^{\varepsilon} \Phi_{j, k+l}^{\varepsilon}(x) \Phi_{j, k}^{\varepsilon}(x) \\
&+\sum_{j \in \mathbb{N}, k \in \mathbb{Z}^{n}} f_{j, k}^{\varepsilon} h_{j, k}^{\varepsilon}\left[\left(\Phi_{j, k}^{\varepsilon}(x)\right)^{2}-2^{j n} \Phi\left(2^{j} x-k\right)\right], \\
& T_{3, \varepsilon}(x)=\sum_{j \in \mathbb{N}, k \in \mathbb{Z}^{n}} \sum_{l \in \mathbb{Z}_{N}^{n} l^{\prime} \in \mathbb{Z}^{n}} f_{j+N, 2^{N} k+l}^{\varepsilon} h_{j, k+l^{\prime}}^{0} \\
& T_{4, \varepsilon}(x)=\sum_{j \in \mathbb{N}, k \in \mathbb{Z}^{n}} f_{j, k}^{\varepsilon} h_{j, k}^{\varepsilon} 2^{j n} \Phi\left(2^{j} x-k\right), \\
& T_{5}(x)=\sum_{k \in \mathbb{Z}^{n}} \sum_{l \in \mathbb{Z}^{n}} f_{0, k}^{0} h_{0, k+l}^{0} \Phi_{j, k}^{0}(x) \Phi_{j, k+l}^{\varepsilon}(x) .
\end{aligned}
$$

For $\varepsilon, \varepsilon^{\prime} \in E_{n}, 0<s \leq N$ or $s=0$, and $\varepsilon \neq \varepsilon^{\prime}$, denote

$$
T_{2, s, \varepsilon, \varepsilon^{\prime}}(x)=\sum_{j \in \mathbb{N}, k \in \mathbb{Z}^{n}} \sum_{l \in \mathbb{Z}^{n}} f_{j+s, 2^{s} k+l}^{\varepsilon^{\prime}} h_{j, k}^{\varepsilon} \Phi_{j+s, 2^{s} k+l}^{\varepsilon^{\prime}}(x) \Phi_{j, k}^{\varepsilon}(x) .
$$

By (11) and (12), we can decompose $f h$ as follows:

$$
\begin{aligned}
f(x) h(x)= & \sum_{\varepsilon \in E_{n}} T_{1, \varepsilon}(x)+\sum_{0 \leq s \leq N, \varepsilon, \varepsilon^{\prime} \in E_{n}} T_{2, s, \varepsilon, \varepsilon^{\prime}}(x) \\
& +\sum_{\varepsilon \in E_{n}} T_{3, \varepsilon}(x)+\sum_{\varepsilon \in E_{n}} T_{4, \varepsilon}(x)+T_{5}(x) \\
:= & \sum_{i=1}^{5} T_{i}(x) .
\end{aligned}
$$

If $g \in H^{t+r, p}\left(\mathbb{R}^{n}\right)$, write $g(x)=\sum_{(\varepsilon, j, k) \in \Lambda_{n}} g_{j, k}^{\varepsilon} \Phi_{j, k}^{\varepsilon}(x)$. For $\varepsilon, \varepsilon^{\prime} \in E_{n}$, and $0 \leq s \leq N$, we define

$$
\begin{aligned}
T_{1, \varepsilon} & =\int T_{1, \varepsilon}(x) g(x) d x, \\
T_{2, s, \varepsilon, \varepsilon^{\prime}} & =\int T_{2, s, \varepsilon, \varepsilon^{\prime}}(x) g(x) d x .
\end{aligned}
$$

Let

$$
\begin{aligned}
& T_{1,1, \varepsilon, l}=\sum_{j \in \mathbb{N}, k \in \mathbb{Z}^{n}} \sum_{j \geq j^{\prime} \geq 0, \varepsilon^{\prime}, k^{\prime} \in \mathbb{Z}^{n}}\left|f_{j, k+l}^{0}\right|\left|h_{j, k}^{\varepsilon}\right|\left|a_{j, k, l, j^{\prime}, k^{\prime}}^{\varepsilon, \varepsilon^{\prime}}\right|\left|g_{j^{\prime}, k^{\prime}}^{\varepsilon^{\prime}}\right|, \\
& T_{1,2, \varepsilon, l}=\sum_{j \in \mathbb{N}, k \in \mathbb{Z}^{n}} \sum_{j<j^{\prime}, \varepsilon^{\prime}, k^{\prime} \in \mathbb{Z}^{n}}\left|f_{j, k+l}^{0}\right|\left|h_{j, k}^{\varepsilon}\right|\left|a_{j, k, l, j^{\prime}, k^{\prime}}^{\varepsilon, \varepsilon^{\prime}}\right|\left|g_{j^{\prime}, k^{\prime}}^{\varepsilon^{\prime}}\right|, \\
& T_{2,1, s, \varepsilon, \varepsilon^{\prime}, l} \\
& =\sum_{j \in \mathbb{N}, k \in \mathbb{Z}^{n}} \sum_{j \geq j^{\prime}>0, \varepsilon^{\prime \prime}, k^{\prime} \in \mathbb{Z}^{n}}\left|f_{j+s, 2^{s} k+l}^{\varepsilon^{\prime}}\right|\left|h_{j, k}^{\varepsilon}\right|\left|a_{j, k, l, j^{\prime}, k^{\prime}}^{\varepsilon, \varepsilon^{\prime}, \varepsilon^{\prime \prime}, s}\right|\left|g_{j^{\prime}, k^{\prime}}^{\varepsilon^{\prime \prime}}\right|, \\
& =\sum_{j \in \mathbb{N}, k \in \mathbb{Z}^{n}} \sum_{j<j^{\prime}, \varepsilon^{\prime \prime}, k}\left|f_{j+s, 2^{s} k+l}^{\varepsilon^{\prime}}\right|\left|h_{j, k}^{\varepsilon}\right|\left|a_{j, k, l, j^{\prime}, k^{\prime} \in \mathbb{Z}^{n}, l}^{\varepsilon, \varepsilon^{\prime \prime}, s}\right|\left|g_{j^{\prime}, k^{\prime}}^{\varepsilon^{\prime \prime}}\right| .
\end{aligned}
$$

It is easy to see that

$$
\begin{gathered}
\left|T_{1, \varepsilon}\right| \lesssim \sum_{l \in \mathbb{Z}^{n}}\left(T_{1,1, \varepsilon, l}+T_{1,2, \varepsilon, l}\right), \\
\left|T_{2, s, \mathcal{\varepsilon}, \mathcal{\varepsilon}^{\prime}}\right| \lesssim \sum_{l \in \mathbb{Z}^{n}}\left(T_{2,1, s, \mathcal{\varepsilon}, \mathcal{\varepsilon}^{\prime}, l}+T_{2,2, s, \mathcal{\varepsilon}, \mathcal{\varepsilon}^{\prime}, l}\right) .
\end{gathered}
$$

3.2. Several Technical Lemmas. Now we estimate the quantities $T_{1,1, \varepsilon, l}, T_{1,2, \varepsilon, l}, T_{2,1, s, \varepsilon, \varepsilon^{\prime}, l}$, and $T_{2,2, s, \varepsilon, \varepsilon^{\prime}, l}$ separately. Let

$$
S_{t} g_{j^{\prime}}(x)=\sum_{\varepsilon^{\prime}, k^{\prime}} 2^{j^{\prime}(n / 2+t)}\left|g_{j^{\prime}, k^{\prime}}^{\varepsilon^{\prime}}\right| \chi\left(2^{j^{\prime}} x-k^{\prime}\right) .
$$

For fixed $x$, there is only one $k^{\prime}$ such that $\chi\left(2^{j^{\prime}} x-k^{\prime}\right) \neq 0$ and the number of $\varepsilon^{\prime}$ is finite. Then

$$
S_{t} g_{j^{\prime}}(x)=\left(\sum_{\varepsilon^{\prime}, k^{\prime}} 2^{2 j^{\prime}(n / 2+t)}\left|g_{j^{\prime}, k^{\prime}}^{\varepsilon^{\prime}}\right|^{2} \chi\left(2^{j^{\prime}} x-k^{\prime}\right)\right)^{1 / 2} .
$$

Let $M$ be the Hardy-Littlewood maximal function. Then, if $j<j^{\prime}$ and $x \in Q_{j, k}$, we have

$$
\sum_{k^{\prime}}\left(1+\left|k-2^{j-j^{\prime}} k^{\prime}\right|\right)^{-N_{2}} 2^{j^{\prime}(-n / 2+t)} g_{j^{\prime}, k^{\prime}}^{\varepsilon^{\prime}} \lesssim 2^{-j n} M S_{t} g_{j^{\prime}}(x)
$$

Lemma 18. Given $r>0, t \geq 0$, and $t+r<1<p<n /(t+r)$. If $f \in B M O^{r}\left(\mathbb{R}^{n}\right)$, then

(i) for $g \in H^{t+r, p}$ and $h \in H^{-t, p^{\prime}}$,

$$
\left|T_{1, \varepsilon}\right|+\left|T_{2, s, \varepsilon, \varepsilon^{\prime}}\right| \leqslant\|g\|_{H^{t+r, p}}\|h\|_{H^{-t, p^{\prime}}}
$$

(ii) for $g \in H^{t+r, p}$ and $h \in H^{-t, p^{\prime}}$,

$$
\left|\left\langle T_{5}, g\right\rangle\right| \lesssim\|g\|_{H^{t+r, p}}\|h\|_{H^{-t, p^{\prime}}}
$$


Proof. (1) For $j^{\prime} \leq j$, we have

$$
T_{1,1, \varepsilon, l}=\sum_{j, k} \sum_{j \geq j^{\prime}, \varepsilon^{\prime}, k^{\prime}}\left|f_{j, k+l}^{0}\right|\left|h_{j, k}^{\varepsilon}\right|\left|a_{j, k, l, j^{\prime}, k^{\prime}}^{\varepsilon, \varepsilon^{\prime}}\right|\left|g_{j^{\prime}, k^{\prime}}^{\varepsilon^{\prime}}\right| .
$$

By Lemma 17, we know

$$
\left|a_{j, k, l, j^{\prime}, k^{\prime}}^{\varepsilon, \varepsilon^{\prime}}\right| \lesssim(1+|l|)^{-N_{1}}\left(1+\left|k^{\prime}-2^{j^{\prime}-j} k\right|\right)^{-N_{2}} 2^{n j^{\prime} / 2+j^{\prime}-j} .
$$

By Lemma 9, $f \in \mathrm{BMO}^{r}\left(\mathbb{R}^{n}\right)$ implies $\left|f_{j, k}^{\varepsilon}\right| \leq C 2^{(r-n / 2) j}$. Now we can get

$$
\begin{aligned}
& \sum_{l \in \mathbb{Z}^{n}} T_{1,1, \varepsilon, l} \lesssim \sum_{l \in \mathbb{Z}^{n}} \sum_{k, j \geq j^{\prime}} \int 2^{3 n j^{\prime} / 2+\left(j^{\prime}-j\right)+(r-n / 2) j} \sum_{k^{\prime}}\left(\sum_{\varepsilon^{\prime}}\left|g_{j^{\prime}, k^{\prime}}^{\varepsilon^{\prime}}\right|^{2}\right)^{1 / 2} \\
& \times \frac{\left|h_{j, k}^{\varepsilon}\right| \chi\left(2^{j^{\prime}} x-k^{\prime}\right)}{(1+|l|)^{N_{1}}\left(1+\left|k^{\prime}-2^{j^{\prime}-j} k\right|\right)^{N_{2}}} d x \\
& \lesssim \sum_{j \geq j^{\prime}} \int^{3 n j^{\prime} / 2+\left(j^{\prime}-j\right)+(r-n / 2) j} \\
& \times \sum_{k^{\prime}}\left(\sum_{\varepsilon^{\prime}} 2^{2 j^{\prime}(n / 2+t+r)}\left|g_{j^{\prime}, k^{\prime}}^{\varepsilon^{\prime}}\right|^{2}\right)^{1 / 2} 2^{-j^{\prime}(t+r+n / 2)} \\
& \times \sum_{k} \frac{\left|h_{j, k}^{\varepsilon}\right| \chi\left(2^{j^{\prime}} x-k^{\prime}\right)}{\left(1+\left|k^{\prime}-2^{j^{\prime}-j} k\right|\right)^{N_{2}} 2^{j(-n / 2-t)} 2^{j(n / 2+t)} d x} \\
& \lesssim \sum_{j \geq j^{\prime}} 2^{3 n j^{\prime} / 2-j^{\prime}(t+r+n / 2)+\left(j^{\prime}-j\right)+(r-n / 2) j+j(n / 2+t)} \\
& \times 2^{-j^{\prime} n} S_{t+r} g_{j^{\prime}}(x) M\left(S_{-t} h_{j}\right)(x) d x .
\end{aligned}
$$

Because $0<t+r<1$,

$$
\begin{aligned}
\sum_{l \in \mathbb{Z}^{n}} T_{1,1, \varepsilon, l} \lesssim & \sum_{j \geq j^{\prime}} 2^{\left(j^{\prime}-j\right)+(r+t) j-j^{\prime}(r+t)} \\
& \times \int S_{t+r} g_{j^{\prime}}(x) M\left(S_{-t} h_{j}\right)(x) d x \\
\lesssim & \sum_{j \geq j^{\prime}} 2^{\left(j^{\prime}-j\right)(1-r-t)}\|g\|_{H^{t+r, p}}\|h\|_{H^{-t, p^{\prime}}} \\
\lesssim & \|g\|_{H^{t+r, p}}\|h\|_{H^{-t, p^{\prime}}} .
\end{aligned}
$$

Now we estimate the term

$$
T_{2,1, s, \varepsilon, \varepsilon^{\prime}, l}(x)=\sum_{j, k} \sum_{j \geq j^{\prime}>0}\left|f_{j, k}^{\varepsilon^{\prime}}\right|\left|h_{j+s, 2^{s} k+l}^{\varepsilon}\right|\left|a_{j, k, l, j^{\prime}, k^{\prime}}^{\varepsilon, \varepsilon^{\prime}, \varepsilon^{\prime \prime}, s}\right|\left|g_{j^{\prime}, k^{\prime}}^{\varepsilon^{\prime \prime}}\right| .
$$

Because $f \in \operatorname{BMO}^{r}\left(\mathbb{R}^{n}\right)$, by Lemma 9, we have $\left|f_{j, k+l}^{\varepsilon^{\prime}}\right| \lesssim$ $2^{(r-n / 2) j}$. By Lemma 17,

$$
\left|a_{j, k, \varepsilon^{\prime}, \varepsilon^{\prime}, k^{\prime}}^{\varepsilon, s}\right| \leqslant(1+|l|)^{-N_{1}}\left(1+\left|k^{\prime}-2^{j^{\prime}-j} k\right|\right)^{-N_{2}} 2^{n j^{\prime} / 2+\left(j^{\prime}-j\right)} .
$$

We can get, similarly,

$$
\begin{aligned}
& \sum_{l \in \mathbb{Z}_{N}^{n}} T_{2,1, s, \varepsilon, \varepsilon^{\prime}, l}(x) \\
& \lesssim \sum_{l \in \mathbb{Z}_{N}^{n}} \sum_{j, k} \sum_{j \geq j^{\prime}>0} 2^{(r-n / 2) j} 2^{n j^{\prime} / 2+j^{\prime}-j} \\
& \times \frac{\left|h_{j+s, 2^{s} k+l}^{\varepsilon}\right|\left|g_{j^{\prime}, k^{\prime}}^{\varepsilon^{\prime \prime}}\right|}{(1+|l|)^{N_{1}}\left(1+\left|k^{\prime}-2^{j^{\prime}-j} k\right|\right)^{N_{2}}} \\
& \lesssim \sum_{l \in \mathbb{Z}_{N}^{n}} \sum_{j \geq j^{\prime}>0} \int 2^{3 n j^{\prime} / 2+\left(j^{\prime}-j\right)+(r-n / 2) j} \\
& \times \sum_{\varepsilon^{\prime \prime}, k^{\prime}} \frac{\left|g_{j^{\prime}, k^{\prime}}^{\varepsilon^{\prime}}\right|\left|h_{j+s, 2^{s} k+l}^{\varepsilon}\right| \chi\left(2^{j^{\prime}} x-k^{\prime}\right)}{(1+|l|)^{N_{1}}\left(1+\left|k^{\prime}-2^{j^{\prime}-j} k\right|\right)^{N_{2}}} d x \\
& \lesssim \sum_{j \geq j^{\prime}>0} 2^{\left(j^{\prime}-j\right)(1-t-r)} \int S_{t+r}\left(g_{j^{\prime}}\right)(x) M S_{-t} h_{j+s}(x) d x \\
& \lesssim\left\|S_{t+r} g_{j^{\prime}}\right\|_{L^{p}}\left\|M S_{-t} h_{j+s}\right\|_{L^{p^{\prime}}} \\
& \lesssim\|g\|_{H^{t+r, p}}\|h\|_{H^{-t, p^{\prime}}} .
\end{aligned}
$$

(2) If $j^{\prime}>j$, the estimates of $T_{1,2, \varepsilon, l}$ and $T_{2,2, s, \varepsilon, \mathcal{E}^{\prime}, l}$ are easier than those of $T_{1,1, \varepsilon, l}$ and $T_{2,1, s, \varepsilon, \varepsilon^{\prime}, l}$. For example, we estimate the term

$$
T_{1,2, \varepsilon, l}=\sum_{j, k} \sum_{0 \leq j<j^{\prime}, \varepsilon^{\prime}, k^{\prime}}\left|f_{j, k+l}^{0}\right|\left|h_{j, k}^{\varepsilon}\right|\left|a_{j, k, l, j^{\prime}, k^{\prime}}^{\varepsilon, \varepsilon^{\prime}}\right|\left|g_{j^{\prime}, k^{\prime}}^{\varepsilon^{\prime}}\right|
$$

Because $f \in \operatorname{BMO}^{r}\left(\mathbb{R}^{n}\right)$, by Lemma $9,\left|f_{j, k+l}^{0}\right| \lesssim 2^{(r-n / 2) j}$. By Lemma 17,

$$
\begin{aligned}
& \mid \begin{array}{l}
a_{j, k, l, j^{\prime}, k^{\prime}}^{\varepsilon, \varepsilon^{\prime}} \\
x^{\prime}
\end{array} \\
& \quad \leq(1+|l|)^{-N_{1}}\left(1+\left|k-2^{j-j^{\prime}} k^{\prime}\right|\right)^{-N_{2}} 2^{-n j^{\prime} / 2+n j+N\left(j-j^{\prime}\right)} .
\end{aligned}
$$


Because $2^{n j} \int \chi_{j, k}(x) d x=1$, we can obtain

$$
\begin{aligned}
& \sum_{l \in \mathbb{Z}^{n}} T_{1,2, \varepsilon, l} \\
& \lesssim \sum_{l \in \mathbb{Z}^{n}} \sum_{j, k} \sum_{0 \leq j<j^{\prime}, \varepsilon^{\prime}, k^{\prime}} \int 2^{(r-n / 2) j} 2^{-n j^{\prime} / 2+n j+N\left(j-j^{\prime}\right)} 2^{n j} \\
& \times \frac{\left|h_{j, k}^{\varepsilon}\right|\left|g_{j^{\prime}, k^{\prime}}^{\varepsilon^{\prime}}\right| \chi_{j, k}(x)}{(1+|l|)^{N_{1}}\left(1+\left|k-2^{j-j^{\prime}} k^{\prime}\right|\right)^{N_{2}}} d x \\
& \lesssim \sum_{\varepsilon, j, k} \int 2^{(r-n / 2)} 2^{-n j^{\prime} / 2+n j+N\left(j-j^{\prime}\right)} 2^{n j} 2^{-n j} 2^{-j^{\prime}(-n / 2+t+r)} \\
& \times M S_{t+r}\left(g_{j^{\prime}}\right)(x)\left|h_{j, k}^{\varepsilon}\right| \chi_{j, k}(x) d x \\
& \lesssim \sum_{0 \leq j<j^{\prime}} \int 2^{(r-n / 2) j} 2^{-\left(n j^{\prime} / 2\right)+n j+N\left(j-j^{\prime}\right)} 2^{-j(n / 2-t)} \\
& \times 2^{-j^{\prime}(-n / 2+t+r)} M S_{t+r}\left(g_{j^{\prime}}\right)(x) S_{-t}\left(h_{j}\right)(x) d x \\
& \lesssim\|g\|_{H^{t+r, p}}\|h\|_{H^{-t, p^{\prime}}} .
\end{aligned}
$$

The estimate for $T_{2,2, s, \mathcal{\varepsilon}, \mathcal{\varepsilon}^{\prime}, l}(x)$ can be obtained similarly. By the same methods used in (1) and (2), we can get the estimate of the term $T_{5}$. We omit the details. claim.

Now we consider the term $T_{3, \varepsilon}$. We have the following

Lemma 19. Given $r>0, t \geq 0$, and $t+r<1<p<n /(t+r)$, if $f \in B M O^{r}\left(\mathbb{R}^{n}\right)$, then

$$
\left|\left\langle T_{3, \mathcal{\varepsilon}}, g\right\rangle\right| \lesssim\|g\|_{H^{t+r, p}}\|h\|_{H^{-t, p^{\prime}}}
$$

Proof. In fact, for $l \in \mathbb{Z}_{N}^{n},(\varepsilon, j, k) \in \Lambda_{n}$ and $l^{\prime} \in \mathbb{Z}^{n}$, let

$$
g_{j+N, 2^{N} k+l}^{\varepsilon, l^{\prime}}=2^{-n j / 2}\left\langle\Phi_{j, k+l^{\prime}}^{0} \Phi_{j+N, 2^{N} k+l}^{\varepsilon}, g\right\rangle
$$

We have

$$
\begin{aligned}
& \left|\left\langle T_{3, \varepsilon}, g\right\rangle\right| \\
& =\left|\sum_{(\varepsilon, j, k) \in \Lambda_{n}} \sum_{l \in \mathbb{Z}_{N}^{n}, l^{\prime} \in \mathbb{Z}^{n}} 2^{j n / 2} h_{j, k+l^{\prime}}^{0} g_{j+N, 2^{N} k+l}^{\varepsilon, l^{\prime}} f_{j+N, 2^{N} k+l}^{\varepsilon}\right| \\
& \quad \lesssim \sum_{l \in \mathbb{Z}_{N}^{n}} \sum_{(\varepsilon, j, k) \in \Lambda_{n}}\left(\sum_{l^{\prime} \in \mathbb{Z}^{n}} 2^{j n / 2}\left|h_{j, k+l^{\prime}}^{0}\right|\left|g_{j+N, 2^{N} k+l}^{\varepsilon, l^{\prime}}\right|\right) \\
& \quad \times\left|f_{j+N, 2^{N} k+l}^{\varepsilon}\right| .
\end{aligned}
$$

Because $\left(F_{1}^{r, 2}\left(\mathbb{R}^{n}\right)\right)^{\prime}=\mathrm{BMO}^{r}\left(\mathbb{R}^{n}\right)$, by Lemmas 9 and 13 , we have

$$
\begin{aligned}
& \left|\left\langle T_{3, \varepsilon}, g\right\rangle\right| \\
& \lesssim \sum_{l \in \mathbb{Z}_{N}^{n}}\|f\|_{\mathrm{BMO}^{r}} \int\left(\sum_{(\varepsilon, j, k) \in \Lambda_{n}} 2^{j(n+2 r+t)}\right. \\
& \times\left(\sum_{l^{\prime} \in \mathbb{Z}^{n}} 2^{j(n / 2-t)}\left|h_{j, k+l^{\prime}}^{0}\right|\left|g_{j+N, 2^{N} k+l}^{\varepsilon, l^{\prime}}\right|\right)^{2} \\
& \left.\times \chi_{j, k}(x)\right)^{1 / 2} d x \\
& \lesssim\|f\|_{\mathrm{BMO}^{r}} \int\left(\sum_{(\varepsilon, j, k) \in \Lambda_{n}} 2^{j(n+2 r+2 t)}\right. \\
& \times\left(\sum_{l^{\prime} \in \mathbb{Z}^{n}}\left(1+\left|l^{\prime}\right|\right)^{N+n}\left|g_{j+N, 2^{N} k+l}^{\varepsilon, l^{\prime}}\right|\right)^{2} \\
& \left.\times \chi_{j, k}(x)\right)^{1 / 2} M T^{t} h(x) d x .
\end{aligned}
$$

Because

$$
\begin{aligned}
& \left|g_{j+N, 2^{N} k+l}^{\varepsilon, l^{\prime}}\right| \\
& =2^{-n j / 2}\left|\left\langle\Phi_{j, k+l^{\prime}}^{0} \Phi_{j+N, 2^{N} k+l^{\prime}}^{\varepsilon} \sum_{\left(\varepsilon^{\prime}, j^{\prime}, k^{\prime}\right) \in \Lambda_{n}} g_{j^{\prime}, k^{\prime}}^{\varepsilon^{\prime}} \Phi_{j^{\prime}, k^{\prime}}^{\varepsilon^{\prime}}\right\rangle\right| \\
& =2^{-n j / 2} \mid\left\langle\Phi_{j, k+l^{\prime}}^{0} \Phi_{j+N, 2^{N} k+l^{\prime}}^{\varepsilon}\right. \\
& \left.\times \sum_{\left(\varepsilon^{\prime}, j^{\prime}, k^{\prime}\right) \in \Lambda_{n},\left|j^{\prime}-j-N\right| \leq 2} g_{j^{\prime}, k^{\prime}}^{\varepsilon^{\prime}} \Phi_{j^{\prime}, k^{\prime}}^{\varepsilon^{\prime}}\right\rangle \mid
\end{aligned}
$$

we can get

$$
\begin{aligned}
& \left(\sum_{(\varepsilon, j, k) \in \Lambda_{n}} 2^{(n+2 t+2 t) j}\right. \\
& \left.\quad \times\left(\sum_{l^{\prime} \in \mathbb{Z}^{n}}\left(1+\left|l^{\prime}\right|\right)^{N+n}\left|g_{j+N, 2^{N} k+l}^{\varepsilon, l^{\prime}}\right|\right)^{2} \chi_{j, k}(x)\right)^{1 / 2} \\
& \lesssim \sum_{\left|j^{\prime}-j-N\right| \leq 2} M S_{t+r} g_{j^{\prime}}(x) .
\end{aligned}
$$


Hence, we have

$$
\begin{aligned}
& \int\left(\sum_{(\varepsilon, j, k) \in \Lambda_{n}} 2^{j(n+2 r+t)}\right. \\
& \left.\quad \times\left(\sum_{l^{\prime} \in \mathbb{Z}^{n}} 2^{j(n / 2-t)}\left|h_{j, k+l^{\prime}}^{0} \| g_{j+N, 2^{\varepsilon} k+l}^{\varepsilon, l^{\prime}}\right|\right)^{2} \chi_{j, k}(x)\right)^{p / 2} d x \\
& \quad \int\left(\sum_{j \geq 0}\left(\sum_{\left|j^{\prime}-j-N\right| \leq 2} M S_{t+r} g_{j^{\prime}}(x)\right)^{2}\right)^{p / 2} d x \leqslant\|g\|_{H^{t+r, p} .}
\end{aligned}
$$

Finally, we obtain, by Hölder's inequality,

$$
\begin{aligned}
\left|\left\langle T_{3, \varepsilon}, g\right\rangle\right| & \lesssim\|f\|_{\mathrm{BMO}^{r}}\|g\|_{H^{t+r, p}}\left\|M T^{t} h\right\|_{p^{\prime}} \\
& \lesssim\|f\|_{\mathrm{BMO}^{r}}\|g\|_{H^{t+r, p}}\|h\|_{H^{-t, p^{\prime}}} .
\end{aligned}
$$

This completes the proof of Lemma 19.

In order to deal with the term

$$
T_{4, \varepsilon}(x)=\sum_{(\varepsilon, j, k) \in \Lambda_{n}} f_{j, k}^{\varepsilon} h_{j, k}^{\varepsilon} 2^{j n} \Phi\left(2^{j} x-k\right)
$$

we need the following estimate.

Lemma 20. Given $r>0, t \geq 0$ with $t+r<1<p<n /(r+t)$, $f \in S_{r, p}^{\Phi, t}\left(\mathbb{R}^{n}\right)$ if and only if

$$
\left|\left\langle T_{4, \mathcal{E}}, g\right\rangle\right| \lesssim\|g\|_{H^{r+t, p}}\|h\|_{H^{-t, p^{\prime}}}
$$

Proof. In fact, for $g \in H^{r+t, p}\left(\mathbb{R}^{n}\right)$,

$$
\left\langle T_{4, \varepsilon}, g\right\rangle=\sum_{(\varepsilon, j, k) \in \Lambda_{n}} f_{j, k}^{\varepsilon} g_{j, k}^{\Phi} h_{j, k}^{\varepsilon}
$$

By Lemma 4, using Hölder's inequality, we have

$$
\begin{aligned}
& \left|\left\langle T_{4, \varepsilon}, g\right\rangle\right| \\
& \lesssim \sum_{(\varepsilon, j, k) \in \Lambda_{n}, j \geq 0} 2^{j n}\left|g_{j, k}^{\Phi}\right|^{2}\left|f_{j, k}^{\varepsilon}\right|^{2} \chi_{j, k}(x) d x \\
& \lesssim \int\left(\sum_{(\varepsilon, j, k) \in \Lambda_{n}, j \geq 0} 2^{j(n+2 t)}\left|g_{j, k}^{\Phi}\right|^{2}\left|f_{j, k}^{\varepsilon}\right|^{2} \chi_{j, k}(x)\right)^{1 / 2}
\end{aligned}
$$

$$
\begin{aligned}
& \times\left(\sum_{(\varepsilon, j, k) \in \Lambda_{n}, j \geq 0} 2^{j(n-2 t)}\left|h_{j, k}^{\varepsilon}\right|^{2} \chi_{j, k}(x)\right)^{1 / 2} d x \\
& \leq\left(\int \left(\sum_{(\varepsilon, j, k) \in \Lambda_{n}, j \geq 0} 2^{j(n+2 t)}\left|g_{j, k}^{\Phi}\right|^{2}\left|f_{j, k}^{\varepsilon}\right|^{2}\right.\right. \\
& \left.\left.\left.\left.\left.\times \chi_{j, k}(x)\right)^{p / 2} d x\right)^{1 / p}\right)^{p^{\prime} / 2}\right)^{1 / p^{\prime}} d x\right)^{2} \\
& \quad \times\left(\int_{(\varepsilon, j, k) \in \Lambda_{n}, j \geq 0} 2^{j(n-2 t)}\left|h_{j, k}^{\varepsilon}\right|^{2} \chi_{j, k}(x)\right)_{S_{r, p}^{\Phi, t}\|h\|_{H^{-t, p^{\prime}} \cdot}}
\end{aligned}
$$

Because $f \in S_{r, p}^{\Phi, t}\left(\mathbb{R}^{n}\right)$, we can see that $\left|\left\langle T_{4, \varepsilon}, g\right\rangle\right| \lesssim$ $\|g\|_{H^{t+r, p}}\|h\|_{H^{-t, p^{\prime}}}$.

Conversely, let

$$
\begin{gathered}
\tau_{j, k}^{\varepsilon}=2^{j t} f_{j, k}^{\varepsilon} g_{j, k}^{\Phi}, \\
\tau(x)=\sum_{(\varepsilon, j, k) \in \Lambda_{n}, j \geq 0} \tau_{j, k}^{\varepsilon} \Phi_{j, k}^{\varepsilon}(x) .
\end{gathered}
$$

Denote $h=|\tau|^{p-2} \bar{\tau}$. For $h(x)=\sum_{(\varepsilon, j, k) \in \Lambda_{n}} h_{j, k}^{\varepsilon} \Phi_{j, k}^{\varepsilon}(x)$, we write $h_{t}(x)$ as

$$
h_{t}(x)=\sum_{(\varepsilon, j, k) \in \Lambda_{n}, j \geq 0} 2^{j t} h_{j, k}^{\varepsilon} \Phi_{j, k}^{\varepsilon}(x):=\sum_{(\varepsilon, j, k) \in \Lambda_{n}}\left(h_{t}\right)_{j, k}^{\varepsilon} \Phi_{j, k}^{\varepsilon}(x) .
$$

It is easy to see that $h \in L^{p^{\prime}}\left(\mathbb{R}^{n}\right)$ is equivalent to $h_{t} \in$ $H^{-t, p^{\prime}}\left(\mathbb{R}^{n}\right)$.

By the wavelet characterization of $H^{t, p}\left(\mathbb{R}^{n}\right)$, we get

$$
\begin{aligned}
& \int\left(\sum_{(\varepsilon, j, k) \in \Lambda_{n}, j \geq 0} 2^{j(n+2 t)}\left|g_{j, k}^{\Phi}\right|^{2}\left|f_{j, k}^{\varepsilon}\right|^{2} \chi_{j, k}(x)\right)^{p / 2} d x \\
& =\int\left(\sum_{(\varepsilon, j, k) \in \Lambda_{n}, j \geq 0} 2^{j n}\left|\tau_{j, k}^{\varepsilon}\right|^{2} \chi_{j, k}(x)\right)^{p / 2} d x \\
& =\int|\tau|^{p} d x=\int \tau h d x \\
& =\sum_{(\varepsilon, j, k) \in \Lambda_{n}} 2^{j(t+n / 2)} f_{j, k}^{\varepsilon} g_{j, k}^{\Phi} h_{j, k}^{\varepsilon} \\
& =\sum_{(\varepsilon, j, k) \in \Lambda_{n}} 2^{j n / 2} f_{j, k}^{\varepsilon} g_{j, k}^{\Phi}\left(h_{t}\right)_{j, k}^{\varepsilon} .
\end{aligned}
$$


Further, we can deduce that

$$
\begin{aligned}
& \int\left(\sum_{(\varepsilon, j, k) \in \Lambda_{n}, j \geq 0} 2^{j(n+2 t)}\left|g_{j, k}^{\Phi}\right|^{2}\left|f_{j, k}^{\varepsilon}\right|^{2} \chi_{j, k}(x)\right)^{p / 2} d x \\
& \quad=\left|\left\langle T_{4, \varepsilon}, g\right\rangle\right| \\
& \quad \leq\|g\|_{H^{t+r, p}}\left\|h_{t}\right\|_{H^{-t, p^{\prime}}} \\
& \quad \leq\|g\|_{H^{t+r, p}}\|\tau\|_{L^{p}}^{p / p^{\prime}} .
\end{aligned}
$$

Hence $\|\tau\|_{L^{p}}<\infty$ and $f \in S_{r, p}^{\Phi, t}\left(\mathbb{R}^{n}\right)$. This completes the proof of this lemma.

\subsection{Wavelet Characterization of Sobolev Multipliers}

Theorem 21. Let $t \geq 0, r>0$ with $t+r<1<p<n /(t+r)$. Then,

$$
X_{r, p}^{t}\left(\mathbb{R}^{n}\right)=M_{r, p}^{t}\left(\mathbb{R}^{n}\right) \cap S_{r, p}^{\Phi, t}\left(\mathbb{R}^{n}\right) .
$$

Proof. We first assume $f \in M_{r, p}^{t}\left(\mathbb{R}^{n}\right) \cap S_{r, p}^{\Phi, t}\left(\mathbb{R}^{n}\right)$. Let $g \in$ $H^{t+r, p}\left(\mathbb{R}^{n}\right)$ and $h \in H^{-t, p^{\prime}}\left(\mathbb{R}^{n}\right)$. By the decomposition given in Section 3.1, we have

$$
\begin{aligned}
f(x) h(x)= & \sum_{\varepsilon \in E_{n}} T_{1, \varepsilon}(x)+\sum_{0 \leq s \leq N, \varepsilon, \varepsilon^{\prime} \in E_{n}} T_{2, s, \varepsilon, \varepsilon^{\prime}}(x) \\
& +\sum_{\varepsilon \in E_{n}} T_{3, \varepsilon}(x)+\sum_{\varepsilon \in E_{n}} T_{4, \varepsilon}(x)+T_{5}(x) .
\end{aligned}
$$

Notice that $f \in M_{r, p}^{t}\left(\mathbb{R}^{n}\right)$ implies that $f \in \mathrm{BMO}^{r}\left(\mathbb{R}^{n}\right)$. By Lemmas 18-20, we obtain that, for $f \in M_{r, p}^{t}\left(\mathbb{R}^{n}\right) \cap S_{r, p}^{\Phi, t}\left(\mathbb{R}^{n}\right)$, $f h \in H^{-t-r, p^{\prime}}\left(\mathbb{R}^{n}\right)$ and

$$
\|f h\|_{H^{-t-r, p^{\prime}}} \leqslant\|f\|_{M_{t, p}^{t} \cap S_{t, p}^{\phi, t}}\|h\|_{H^{-t p^{\prime}}},
$$

which gives

$$
\begin{aligned}
& \|f\|_{X_{r, p}^{t}}=\sup _{\|g\|_{H^{r+t, p}} \leq 1} \sup _{\|h\|_{H^{-t, p^{\prime}}}}|\langle f g, h\rangle| \\
& =\sup _{\|g\|_{H^{r+t, p}} \leq 1} \sup _{\|h\|_{H^{-t, p^{\prime}}}}|\langle f h, g\rangle| \\
& \leq \sup _{\|g\|_{H^{r+t, p}} \leq 1} \sup _{\|h\|_{H^{-t, p^{\prime}}}}\|f h\|_{H^{-t-r, p^{\prime}}}\|g\|_{H^{t+r, p}} \\
& \leq\|f\|_{M_{r, p}^{t} \cap S_{r, p}^{\Phi, t}} \text {. }
\end{aligned}
$$

Hence, we can get $M_{r, p}^{t}\left(\mathbb{R}^{n}\right) \cap S_{r, p}^{\Phi, t}\left(\mathbb{R}^{n}\right) \subset X_{r, p}^{t}\left(\mathbb{R}^{n}\right)$.

Conversely, assume that $f \in X_{r, p}^{t}\left(\mathbb{R}^{n}\right)$. By Lemma 7, we have $f \in M_{r, p}^{t}\left(\mathbb{R}^{n}\right) \subset \mathrm{BMO}^{r}\left(\mathbb{R}^{n}\right)$. We apply Lemmas 18 and 19 again to deduce

$$
\begin{aligned}
\left|\left\langle T_{4, \varepsilon}, g\right\rangle\right| & \leq\left|T_{1, \varepsilon}\right|+\left|T_{2, s, \varepsilon, \varepsilon^{\prime}}\right|+\left|\left\langle T_{3, \varepsilon}, g\right\rangle\right|+\left|\left\langle T_{5}, g\right\rangle\right| \\
& \leq\|g\|_{H^{r+t, p}}\|h\|_{H^{-t, p^{\prime}}} .
\end{aligned}
$$

Then, Lemma 20 implies that $f \in S_{r, p}^{\Phi, t}\left(\mathbb{R}^{n}\right)$. This completes the proof of this theorem.
Now we characterize $X_{r, p}^{t}\left(\mathbb{R}^{n}\right)$ by the fractional integration. Let $\Psi(x) \geq 0, \Psi(x) \in C_{0}^{\infty}(B(0,2))$ with $\Psi(x)=1$ on $B(0,1)$. It is well known that $\tilde{g} \in H^{t+r, p}\left(\mathbb{R}^{n}\right)$ if and only if there exists $g \in L^{p}\left(\mathbb{R}^{n}\right)$ such that

$$
\widetilde{g}(x)=J_{t+r} g(x)=\int g(y) \Psi(|x-y|)|x-y|^{t+r-n} d y .
$$

For $g \in L^{p}\left(\mathbb{R}^{n}\right)$, let

$$
g_{j, k}=\int\left(2^{-j}+\left|y-2^{-j} k\right|\right)^{t+r-n} g(y) d y .
$$

Definition 22. Given $r>0, t \geq 0$ and $1<p<n /(r+t)$, then $f(x)=\sum_{(\varepsilon, j, k) \in \Lambda_{n}} f_{j, k}^{\varepsilon} \Phi_{j, k}^{\varepsilon}(x) \in Q_{r, p}^{t}\left(\mathbb{R}^{n}\right)$ if and only if

$$
\int\left(\sum_{(\varepsilon, j, k) \in \Lambda_{n}} 2^{j(n+2 t)}\left|g_{j, k}\right|^{2}\left|f_{j, k}^{\varepsilon}\right|^{2} \chi_{j, k}(x)\right)^{p / 2} d x \leqslant 1
$$

where $g \in L^{p}\left(\mathbb{R}^{n}\right)$ and $g(x) \geq 0$.

Let $f(x)=\sum_{(\varepsilon, j, k) \in \Lambda_{n}} f_{j, k}^{\varepsilon} \Phi_{j, k}^{\varepsilon}(x)$ and $h(x)=$ $\sum_{(\varepsilon, j, k) \in \Lambda_{n}} h_{j, k}^{\varepsilon} \Phi_{j, k}^{\varepsilon}(x)$. Define

$$
T_{\Phi}(f, h)(x)=\sum_{j \in \mathbb{N}} \sum_{k \in \mathbb{Z}^{n}} 2^{j n} f_{j, k}^{\varepsilon} h_{j, k}^{\varepsilon} \Phi\left(2^{j} x-k\right) .
$$

Similar to Lemma 20, we can get the following.

Lemma 23. Given $t \geq 0, r>0$ and $t+r<1<p<n /(t+r)$, then $f \in S_{r, p}^{\Phi, t}\left(\mathbb{R}^{n}\right)$ if and only if

$$
\left|\left\langle T_{\Phi}(f, h), g\right\rangle\right| \lesssim\|g\|_{H^{r+t, p}}\|h\|_{H^{-t, p^{\prime}}}
$$

where $g \in H^{t+r, p}\left(\mathbb{R}^{n}\right)$ and $h \in H^{-t, p^{\prime}}\left(\mathbb{R}^{n}\right)$.

Now we give another characterization of $X_{r, p}^{t}\left(\mathbb{R}^{n}\right)$.

Theorem 24. Given $t \geq 0, r>0$ and $t+r<1<p<n /(t+r)$, then

$$
X_{r, p}^{t}\left(\mathbb{R}^{n}\right)=M_{r, p}^{t}\left(\mathbb{R}^{n}\right) \cap Q_{r, p}^{t}\left(\mathbb{R}^{n}\right)
$$

Proof. By modifying the coefficients such that $f_{j, k}^{\varepsilon} h_{j, k}^{\varepsilon}=$ $\left|f_{j, k}^{\varepsilon}\right|\left|h_{j, k}^{\varepsilon}\right|$, we could suppose that $g(y) \geq 0$. By Theorem 21 (ii) and Lemma 23, we know that $f \in S_{r, p}^{\Phi, t}\left(\mathbb{R}^{n}\right)$ is equivalent to

$$
\begin{aligned}
& \iint_{(\varepsilon, j, k) \in \Lambda_{n}}\left|f_{j, k}^{\varepsilon} \| h_{j, k}^{\varepsilon}\right| 2^{j n} \Phi \\
& \quad \times\left(2^{j} x-k\right) \Psi(|x-y|) \frac{g(y)}{|x-y|^{n-(t+r)}} d y d x \\
& \leq\|g\|_{L^{p}}\|h\|_{H^{-t, p^{\prime}}}, \quad \forall g \geq 0 .
\end{aligned}
$$


By a simple calculation, we get

$$
\begin{aligned}
\int 2^{j n} & \Phi\left(2^{j} x-k\right) \Psi(|x-y|)|x-y|^{t+r-n} d x \\
\simeq & \left(2^{-j}+\left|y-2^{-j} k\right|\right)^{(t+r-n)} .
\end{aligned}
$$

So (95) is equivalent to the following inequality:

$$
\begin{aligned}
& \int \sum_{(\varepsilon, j, k) \in \Lambda_{n}}\left|f_{j, k}^{\varepsilon} \| h_{j, k}^{\varepsilon}\right|\left(2^{-j}+\left|y-2^{-j} k\right|\right)^{t+r-n} g(y) d y \\
& \quad \leq\|g\|_{L^{p}}\|h\|_{H^{-t, p^{\prime}}}, \quad \forall g \geq 0 .
\end{aligned}
$$

That is to say

$$
\begin{aligned}
& \iint_{(\varepsilon, j, k) \in \Lambda_{n}} 2^{j n}\left|f_{j, k}^{\varepsilon} \| h_{j, k}^{\varepsilon}\right| \chi_{j, k} \\
& \quad \times(x) \frac{g(y)}{\left(2^{-j}+\left|y-2^{-j} k\right|\right)^{n-(t+r)}} d x d y \\
& \quad \leq\|g\|_{L^{p}}\|h\|_{H^{-t, p^{\prime}}}, \quad \forall g \geq 0 .
\end{aligned}
$$

Let

$$
S_{-t} h(x)=\left(\sum_{(\varepsilon, j, k) \in \Lambda_{n}} 2^{j(n-2 t)}\left|h_{j, k}^{\varepsilon}\right|^{2} \chi_{j, k}(x)\right)^{1 / 2} .
$$

We have $\left\|S_{-t} h\right\|_{p^{\prime}} \simeq e q\|h\|_{H^{-t, p^{\prime}}}$. Then, the inequality (98) is equivalent to

$$
\begin{aligned}
& \int\left(\sum_{(\varepsilon, j, k) \in \Lambda_{n}} 2^{j(n+2 t)}\left|f_{j, k}^{\varepsilon}\right|^{2}\left|g_{j, k}\right|^{2} \chi_{j, k}(x)\right)^{p / 2} d x \\
& \quad \leq\|g\|_{L^{p}}^{p}, \quad \forall g \geq 0 .
\end{aligned}
$$

The above inequality is equivalent to $f \in Q_{r, p}^{t}\left(\mathbb{R}^{n}\right)$.

\section{Logarithmic Morrey Spaces and Multipliers}

4.1. A Logarithmic Condition. Lemma 7 implies that $X_{r, p}^{t}\left(\mathbb{R}^{n}\right) \subset M_{r, p}^{t}\left(\mathbb{R}^{n}\right)$. In this section, we consider the reverse inclusion relation. At first we introduce a logarithmic Morrey spaces.

Definition 25. Fix $1<p<n /(r+t)$ and $\tau \geq 0$. We say $f(x) \in$ $M_{r, p}^{t, \tau}\left(\mathbb{R}^{n}\right)$ if $\sup _{|\mathrm{Q}|=1}\left|f_{t, \mathrm{Q}}\right| \leq C$ and

$$
\int_{\mathrm{Q}}\left|(I-\Delta)^{t / 2} f(x)-f_{t, \mathrm{Q}}\right|^{p} d x \leqq\left|1-\log _{2}\right| Q||^{-p \tau}|Q|^{1-p(r+t) / n},
$$

for any cube $Q$ with $|Q| \leq 1$.

Similar to Theorem 6, we have the following wavelet characterization of $M_{r, p}^{t, \tau}\left(\mathbb{R}^{n}\right)$.
Theorem 26. Given $t, \tau \geq 0, r>0,1<p<n /(r+t), f(x)=$ $\sum_{(\varepsilon, j, k) \in \Lambda_{n}} f_{j, k}^{\varepsilon} \Phi_{j, k}^{\varepsilon}(x)$ belongs to $M_{r, p}^{t, \tau}\left(\mathbb{R}^{n}\right)$ if and only if

$$
\begin{gathered}
\int_{Q}\left(\sum_{\varepsilon \in E_{n}, Q_{j, k} \subset Q} 2^{j(n+2 t)}\left|f_{j, k}^{\varepsilon}\right|^{2} \chi_{j, k}(x)\right)^{p / 2} d x \\
\lesssim\left(1-\log _{2}|Q|\right)^{-p \tau}|Q|^{1-p(r+t) / n},
\end{gathered}
$$

where $Q \in \Omega$ with $|Q| \leq 1$.

Proof. Similar to that of Theorem 6, the proof of this theorem can be obtained by the characterization of Triebel-Lizorkin spaces. See Lemma 4. We omit the detail.

In [8], Fefferman established the following relation:

$$
M_{r, q}^{t}\left(\mathbb{R}^{n}\right) \subset X_{r, p}^{t}\left(\mathbb{R}^{n}\right) \subset M_{r, p}^{t}\left(\mathbb{R}^{n}\right),
$$

where $q>p>1$. In this section, we use wavelet characterization to give a logarithmic type inclusion. Let $r>0, t \geq 0$, $1<p<n /(r+t)$, and $\tau>1 / p^{\prime}$. In Theorem 32, we prove that $M_{r, p}^{t, \tau}\left(\mathbb{R}^{n}\right)$ is a subspace of $X_{r, p}^{t}\left(\mathbb{R}^{n}\right)$. Hence,

$$
M_{r, p}^{t, \tau}\left(\mathbb{R}^{n}\right) \subset X_{r, p}^{t}\left(\mathbb{R}^{n}\right) \subset M_{r, p}^{t}\left(\mathbb{R}^{n}\right)=M_{r, p}^{t, 0}\left(\mathbb{R}^{n}\right) .
$$

Lemma 27. Given $\tau>0, r>0, t \geq 0$, and $1<p<n /(r+t)$, if $f \in M_{r, p}^{t, \tau}\left(\mathbb{R}^{n}\right)$, then

$$
\left|f_{j, k}^{\varepsilon}\right| \lesssim 2^{j(r-n / 2)}(1+j)^{-\tau} .
$$

Proof. Because $f \in M_{r, p}^{t, \tau}\left(\mathbb{R}^{n}\right)$, then for $Q \in \Omega$,

$$
\begin{gathered}
\int_{Q}\left(\sum_{\varepsilon \in E_{n}, Q_{j, k} \subset Q} 2^{j(n+2 t)}\left|f_{j, k}^{\varepsilon}\right|^{2} \chi_{j, k}(x)\right)^{p / 2} d x \\
\leq\left(1-\log _{2}|Q|\right)^{-p \tau}|Q|^{1-p(r+t) / n} .
\end{gathered}
$$

We have

$$
\begin{aligned}
& \int_{Q}\left(\sum_{\varepsilon \in E_{n}, Q_{j, k} \subset Q} 2^{j(n-2 r)}\left|f_{j, k}^{\varepsilon}\right|^{2} \chi_{j, k}(x)\right)^{p / 2} d x \\
& \leq|Q|^{p(r+t) / n} \int_{Q}\left(\sum_{\varepsilon \in E_{n}, Q_{j, k} \subset Q} 2^{j(n+2 t)}\left|f_{j, k}^{\varepsilon}\right|^{2} \chi_{j, k}(x)\right)^{p / 2} d x \\
& \leq|Q|^{p(r+t) / n}|Q|^{1-p(r+t) / n}\left(1-\log _{2}|Q|\right)^{-\tau p} \\
& \leq|Q|\left(1-\log _{2}|Q|\right)^{-\tau p} .
\end{aligned}
$$

For $\varepsilon \in E_{n}, j \in \mathbb{N}$, and $k \in \mathbb{Z}^{n}$, take $Q=Q_{j, k}$. By Lemma 4 , we get

$$
\begin{aligned}
\int\left(2^{j n-2 j r}\left|f_{j, k}^{\varepsilon}\right|^{2} \chi_{j, k}(x)\right)^{p / 2} d x & \lesssim 2^{-j n}\left(1-\log _{2} 2^{-j n}\right)^{-\tau p} \\
& \lesssim 2^{-j n}(1+j)^{-\tau p}
\end{aligned}
$$


which gives

$$
\left|f_{j, k}^{\varepsilon}\right| \lesssim 2^{j(r-n / 2)}(1+j)^{-\tau}
$$

When $\varepsilon=0$,

$$
\begin{aligned}
f_{j, k}^{0} & =\left\langle\sum_{\left(\varepsilon^{\prime}, j^{\prime}, k^{\prime}\right) \in \Lambda_{n}} f_{j^{\prime}, k^{\prime}}^{\varepsilon^{\prime}} \Phi_{j^{\prime}, k^{\prime}}^{\varepsilon^{\prime}}, \Phi_{j, k}^{0}\right\rangle \\
& =\left\langle\sum_{\left(\varepsilon^{\prime}, j^{\prime}, k^{\prime}\right) \in \Lambda_{n}: j^{\prime}<j} f_{j^{\prime}, k^{\prime}}^{\varepsilon^{\prime}} \Phi_{j^{\prime}, k^{\prime}}^{\varepsilon^{\prime}}, \Phi_{j, k}^{0}\right\rangle .
\end{aligned}
$$

Since

$$
\left|\sum_{\left(\varepsilon^{\prime}, j^{\prime}, k^{\prime}\right) \in \Lambda_{n}: j^{\prime}<j} f_{j^{\prime}, k^{\prime}}^{\varepsilon^{\prime}} \Phi_{j^{\prime}, k^{\prime}}^{\varepsilon^{\prime}}(x)\right| \lesssim 2^{(r-n / 2) j}(1+j)^{-\tau},
$$

we have

$$
\left|f_{j, k}^{0}\right| \lesssim\left\langle 2^{r j}(1+j)^{-\tau},\left|\Phi_{j, k}^{0}\right|\right\rangle \lesssim 2^{(r-n / 2) j}(1+j)^{-\tau} .
$$

Let $\beta=\left(\beta_{1}, \beta_{2}, \ldots, \beta_{n}\right), \beta_{i} \in \mathbb{N}_{+}$, where $\mathbb{N}_{+}$denotes the set of all positive integers. Denote by $f_{\beta}(x)$ the derivative $(\partial / \partial x)^{\beta} f$. We have the following two lemmas.

Lemma 28. Given $\tau>0, r>0, t \geq 0$, and $1<p<n /(r+t)$, if $f \in M_{r, p}^{t, \tau}\left(\mathbb{R}^{n}\right)$, the derivative $f_{\beta} \in M_{r+|\beta|, p}^{t-|\beta|, \tau}\left(\mathbb{R}^{n}\right)$, where $|\beta|=$ $\sum_{i=1}^{n} \beta_{i}$.

Proof. If $f \in M_{r, p}^{t, \tau}\left(\mathbb{R}^{n}\right)$ and $f(x)=\sum_{(\varepsilon, j, k) \in \Lambda_{n}} f_{j, k}^{\varepsilon} \Phi_{j, k}^{\varepsilon}(x)$, by Theorem 26, we have

$$
\begin{gathered}
\int\left(\sum_{\varepsilon \in E_{n}, Q_{j, k} \subset Q} 2^{j(n+2 t)}\left|f_{j, k}^{\varepsilon}\right|^{2} \chi_{j, k}(x)\right)^{p / 2} d x \\
\leq\left(1-\log _{2}|Q|\right)^{-p \tau}|Q|^{1-p(r+t) / n} .
\end{gathered}
$$

Denote by $f_{j, k}^{\varepsilon, \beta}$ the wavelet coefficients of $f_{\beta}(x)$. We can get $f_{j, k}^{\beta, \varepsilon}=2^{j|\beta|} f_{j, k}^{\varepsilon}$ and

$$
\begin{gathered}
\int\left(\sum_{\varepsilon \in E_{n}, Q_{j, k} \subset Q} 2^{j(n+2 t-2|\beta|)}\left|f_{j, k}^{\beta, \varepsilon}\right|^{2} \chi_{j, k}(x)\right)^{p / 2} d x \\
\quad \lesssim \int\left(\sum_{\varepsilon \in E_{n}, Q_{j, k} \subset Q} 2^{j(n+2 t)}\left|f_{j, k}^{\varepsilon}\right|^{2} \chi_{j, k}(x)\right)^{p / 2} d x \\
\quad \lesssim\left(1-\log _{2}|Q|\right)^{-p \tau}|Q|^{1-p[(r+|\beta|)+(t-|\beta|)] / n}
\end{gathered}
$$

This implies that $f_{\beta}(x) \in M_{r+|\beta|, p}^{t-|\beta|, \tau}\left(\mathbb{R}^{n}\right)$.
To get the sufficient condition for multiplier spaces, we need to consider carefully the relationship of different dyadic cubes relative to combination atoms. Because of this reason, we use always the same Daubechies wavelets in the rest of this section. For a cube $Q$ and a measurable set $E$, if $|Q \bigcap E|=|Q|$, we say that $Q$ is a cube in the set $E$.

For $\left(m, 2^{u}, E_{u}\right)$-combination atoms $g_{u}(x)$ defined in Definition 14, the measure of $E_{u}$ is finite. Hence, the number of biggest dyadic cubes in $E_{u}$ is finite. We denote the number of biggest dyadic cubes in $E_{u}$ by $i_{1}$. Denote by $F_{u, 1}$ the set $\left\{i \in \mathbb{N}, i=1, \ldots, i_{1}\right\}$. If $i \in F_{u, 1}$, we denote such cube by $Q_{u, 1, i}$. The volume of $Q_{u, 1, i}$ is denoted by $2^{-n j_{u, 1}}$; that is, $\left|Q_{u, 1, i}\right|=2^{-n j_{u, 1}}$. Denote $E_{u, 1}=E_{u} \backslash\left(\bigcup_{i \in F_{u, 1}} Q_{u, 1, i}\right)$.

The measure of $E_{u, 1}$ is finite. We denote the number of biggest dyadic cubes in $E_{u, 1}$ by $i_{2}$. Denote by $F_{u, 2}$ the set $\{i \epsilon$ $\left.\mathbb{N}, i=1, \ldots, i_{2}\right\}$. If $i \in F_{u, 2}$, we denote such cube by $Q_{u, 2, i}$. The volume of $Q_{u, 2, i}$ is denoted by $2^{-n j_{u, 2}}$; that is, $\left|Q_{u, 2, i}\right|=2^{-n j_{u, 2}}$. Denote $E_{u, 2}=E_{u, 1} \backslash\left(\bigcup_{i \in F_{u, 2}} Q_{u, 2, i}\right)$.

We continue this process until there exists some $s$ such that $E_{u, s+1}$ is empty. For $s^{\prime} \geq s+1$, we denote $i_{s^{\prime}}=0$, and $F_{u, s^{\prime}}$ and $E_{u, s^{\prime}}$ are empty sets. Otherwise we continue until infinity. Then $E_{u}=\bigcup_{s \geq 1, i \in F_{u, s}} Q_{u, s, i}$ and $g_{u}(x)=\sum_{s \geq 1, i \in F_{u, s}} g_{u, s, i}(x)$, where $g_{u, s, i}(x)=\sum_{Q_{j, k} \subset Q_{u, s, i}} g_{j, k}^{\varepsilon} \Phi_{j, k}^{\varepsilon}(x)$.

To compute the norm of $f(x) g_{u}(x)$, we need to find out a special set of dyadic cubes denoted by $\left\{Q_{u, s, i, k}\right\}_{(s, i, k) \in H_{u}}$ such that supp $g_{u} \subset \bigcup_{(s, i, k) \in H_{u}} Q_{u, s, i, k} \cdot g_{u}(x)$ is nearly $L^{\infty}$ function on $Q_{u, s, i, k}$ and satisfies the estimate of Lemma 29. We divide such process into the following three steps.

Step 1. For all $s \geq 1$, if $i \in F_{u, s}$ and $k \in \mathbb{Z}^{n}$ with $|k|<$ $\sqrt{n} 2^{(M+3) n}$, we denote $(i, k) \in G_{u, s}$. Denote $F_{u}=\{(s, i, k), s \geq$ $\left.1,(i, k) \in G_{u, s}\right\}$. For $(s, i, k) \in F_{u}$, we denote $Q_{u, s, i, k}=$ $2^{-j_{u, s}} k+Q_{u, s, i}$. For $s \geq 1$, we denote $\widetilde{E}_{u, s}^{0}=\bigcup_{(i, k) \in G_{u, s}} Q_{u, s, i, k}$. In the next step, we choose a special subcover to the support of $g_{u}(x)$.

Step 2. We define now $H_{u, s}(s \geq 1), \widetilde{E}_{u, s}^{1}(s \geq 1)$ and $G_{u, s}^{1}(s \geq$ 2).

For $s=1$, denote $H_{u, 1}=G_{u, 1}$ and $\widetilde{E}_{u, s}^{1}=\widetilde{E}_{u, s}^{0}$. For $s=2$, denote $(i, k) \in G_{u, 2}^{1}$, if there exists $0 \leq j \leq j_{u, 1}, l \in \mathbb{Z}^{n}$ such that $Q_{j, l} \subset \bigcup_{\left|k-k^{\prime}\right| \leq \sqrt{n} 2^{(M+2) n}} Q_{u, 2, i, k^{\prime}}$ and $\left\langle g_{u}, \Phi_{j, l}^{0}(x)\right\rangle \neq 0$. We know that $\bigcup_{(i, k) \in G_{u, 2}^{1}} Q_{u, 2, i, k} \subset \widetilde{E}_{u, 1}^{1}$. Denote $H_{u, 2}=G_{u, 2} \backslash G_{u, 2}^{1}$ and $\widetilde{E}_{u, 2}^{1}=\bigcup_{(i, k) \in H_{u, 2}} Q_{u, 2, i, k}$.

For $s=3$, denote $(i, k) \in G_{u, 3}^{1}$, if there exists $0 \leq$ $j \leq j_{u, 2}, l \in \mathbb{Z}^{n}$ such that $Q_{j, l} \subset \bigcup_{\left|k-k^{\prime}\right| \leq \sqrt{n} 2^{(M+2) n}} Q_{u, 2, i, k^{\prime}}$ and $\left\langle g_{u}(x), \Phi_{j_{u, s}, l}^{0}(x)\right\rangle \neq 0$. We know that $\bigcup_{(i, k) \in G_{u, 3}^{1}} Q_{u, 3, i, k} \subset$ $\bigcup_{1 \leq s \leq 2} \widetilde{E}_{u, s}^{1}$. Denote $H_{u, 3}=G_{u, 3} \backslash G_{u, 3}^{1}$ and $\widetilde{E}_{u, 3}^{1}=$ $\bigcup_{(i, k) \in H_{u, 3}} Q_{u, 3, i, k}$.

We continue this process until infinity. For $s \geq 2$, maybe, a part of $G_{u, s}^{1}, H_{u, s}$, and $\widetilde{E}_{u, s}^{1}$ is empty set.

Step 3. Let $H_{u}=\left\{(s, i, k), s \geq 1,(i, k) \in H_{u, s}\right\}$. It is easy to see that the support of $g_{u}(x)$ is contained in $\bigcup_{s \geq 1} \widetilde{E}_{u, s}^{1}$. For a 
$\left(t+r, 2^{u}, E_{u}\right)$-combination atom $g_{u}(x)$ and $g_{j, k}^{0}=\left\langle g_{u}, \Phi_{j, k}^{0}\right\rangle$, we have the following estimate.

Lemma 29. Given $r>0, t \geq 0,1<p<n /(t+r)$ and $s \geq 1$, for $\left(i, m^{\prime}\right) \in H_{u, s}$ and $Q_{j, k} \subset Q_{u, s, i, m^{\prime}}$, we have $\left|g_{j, k}^{0}\right| \leqslant 2^{u-n j / 2} 2^{-(t+r) j_{u, s}}$.

Proof. By the definition of $H_{u, s}$, we have

$$
\begin{aligned}
g_{j, k}^{0} & =\left\langle\sum_{\left(\varepsilon^{\prime}, j^{\prime}, k^{\prime}\right) \in \Lambda_{n}} g_{j^{\prime}, k^{\prime}}^{\varepsilon^{\prime}} \Phi_{j^{\prime}, k^{\prime}}^{\varepsilon^{\prime}}, \Phi_{j, k}^{0}\right\rangle \\
& =\left\langle\sum_{\left(\varepsilon^{\prime}, j^{\prime}, k^{\prime}\right) \in \Lambda_{n}: j^{\prime} \geq j_{u, s}} g_{j^{\prime}, k^{\prime}}^{\varepsilon^{\prime}} \Phi_{j^{\prime}, k^{\prime}}^{\varepsilon^{\prime}}, \Phi_{j, k}^{0}\right\rangle .
\end{aligned}
$$

Because $g_{u}$ is a $\left(m, 2^{u}, E_{u}\right)$-combination atom, $S_{t+r}\left(g_{u}\right)(x) \leq C 2^{u}$. Hence, for every $\left(\varepsilon^{\prime}, j^{\prime}, k^{\prime}\right)$, $\left|g_{j^{\prime}, k^{\prime}}^{\varepsilon^{\prime}}\right| \lesssim 2^{u} 2^{-j^{\prime}(r+t+n / 2)}$. We can obtain

$$
\begin{aligned}
\left|g_{j, k}^{0}\right|= & \left|\left\langle\sum_{\left(\varepsilon^{\prime}, j^{\prime}, k^{\prime}\right) \in \Lambda_{n}: j^{\prime} \geq j_{u, s}} g_{j^{\prime}, k^{\prime}}^{\varepsilon^{\prime}} \Phi_{j^{\prime}, k^{\prime}}^{\varepsilon^{\prime}}, \Phi_{j, k}^{0}\right\rangle\right| \\
\leqslant & 2^{u} \int \sum_{j^{\prime} \geq j_{u, s}} 2^{-j^{\prime}(r+t)} 2^{-j n^{\prime} / 2} 2^{j^{\prime} n / 2} 2^{j n / 2} \\
& \times\left|\Phi^{\varepsilon^{\prime}}\left(2^{j^{\prime}} x-k^{\prime}\right)\right|\left|\Phi^{0}\left(2^{j} x-k\right)\right| d x \\
& \leqslant 2^{u} 2^{-j n / 2} 2^{-j_{u, s}(r+t)} .
\end{aligned}
$$

Theorem 30. Suppose that $\tau>1 / p^{\prime}, t \geq 0, r>0$, and $1<p<$ $n /(r+t)$. If $f \in M_{r, p}^{t, \tau}\left(\mathbb{R}^{n}\right)$ and $g_{u}$ is $a\left(t+r, 2^{u}, E_{u}\right)$-combination atom, then, for $s \geq 1,\left(i, m^{\prime}\right) \in H_{u, s}$, and $Q=Q_{u, s, i, m^{\prime}}$, we have

$$
\left\|f g_{u}\right\|_{H^{t, p}(\mathrm{Q})} \leqslant\left(1+j_{u, s}\right)^{-\tau} 2^{u}|Q|^{1 / p} .
$$

Proof. First, for $t \geq 0$, we prove $\left\|f g_{u}\right\|_{L^{p}(Q)} \leq C j_{\mathcal{u}, s}^{-\tau} 2^{u}|Q|^{1 / p}$. Let $f(x)=\sum_{(\varepsilon, j, k) \in \Lambda_{n}} f_{j, k}^{\varepsilon} \Phi_{j, k}^{\varepsilon}(x)$ and $g_{u}(x)=$ $\sum_{(\varepsilon, j, k) \in \Lambda_{n}} g_{j, k}^{\varepsilon} \Phi_{j, k}^{\varepsilon}(x)$. Denote by $\Lambda_{n}^{\prime}$ the set

$$
\begin{gathered}
\left\{\left(\varepsilon, \varepsilon^{\prime}, j, k, l\right), \varepsilon, \varepsilon^{\prime} \in E_{n},(\varepsilon, k) \neq\left(\varepsilon^{\prime}, k+l\right),\right. \\
\left.j \in \mathbb{Z}, k, l \in \mathbb{Z}^{n},|l| \leq \sqrt{n} 2^{(M+2) n}\right\} .
\end{gathered}
$$

For any dyadic cube $Q \subset E_{u}$, by the formulas (11) and (12), we decompose $f g_{u}$ as

$$
\begin{aligned}
& T_{1}(x)=\sum_{(\varepsilon, j, k) \in \Lambda_{n}, j=j_{u, s}} \sum_{l l \mid \leq \sqrt{n} 2^{(M+2) n}} f_{j, k+l}^{0} g_{j, k}^{0} \Phi_{j, k+l}^{0}(x) \Phi_{j, k}^{0}(x), \\
& T_{2}(x)=\sum_{(\varepsilon, j, k) \in \Lambda_{n}, j \geq j_{u, s}} \sum_{|l| \leq \sqrt{n} 2^{(M+2) n}} f_{j, k+l}^{0} g_{j, k}^{\varepsilon} \Phi_{j, k+l}^{0}(x) \Phi_{j, k}^{\varepsilon}(x), \\
& T_{3}(x)=\sum_{(\varepsilon, j, k) \in \Lambda_{n}, j \geq j_{u, s}} \sum_{\left(l l \leq \leq \sqrt{n} 2^{(M+2) n}\right.} f_{j, k+l}^{\varepsilon} g_{j, k}^{0} \Phi_{j, k+l}^{\varepsilon}(x) \Phi_{j, k}^{0}(x), \\
& T_{4}(x)=\sum_{\left(\varepsilon, \varepsilon^{\prime}, j, k, l\right) \in \Lambda_{n}^{\prime}, j \geq j_{u, s}|l| \leq \sqrt{n} 2^{(M+2) n}} f_{j, k+l}^{\varepsilon^{\prime}} \\
& T_{5}(x)=\sum_{(\varepsilon, j, k) \in \Lambda_{n}, j \geq j_{u, s}} f_{j, k}^{\varepsilon} \Phi_{j, k+l}^{\varepsilon^{\prime}}(x) \Phi_{j, k}^{\varepsilon}(x), \\
& T_{6}(x)=\sum_{(\varepsilon, j, k) \in \Lambda_{n}, j \geq j_{u, s}} f_{j, k}^{\varepsilon} g_{j, k}^{\varepsilon} 2^{n j / 2} \Phi_{j, k}^{0}(x) .
\end{aligned}
$$

The rest of the proof is divided into three steps.

Step 1. For $t \geq 0$, we estimate $\left\|T_{i}\right\|_{L^{p}(Q)}, i=1,2, \ldots, 6$. By Lemma 4, we obtain

$$
\begin{aligned}
f & =\sum_{(\varepsilon, j, k) \in \Lambda_{n}} f_{j, k}^{\varepsilon} \Phi_{j, k}^{\varepsilon}(x) \in L^{p} \\
& \Longleftrightarrow\left\|\left(\sum_{(\varepsilon, j, k) \in \Lambda_{n}} 2^{j n}\left|f_{j, k}^{\varepsilon}\right|^{2} \chi\left(2^{j} \cdot-k\right)\right)^{1 / 2}\right\|_{L^{p}} .
\end{aligned}
$$

Let

$$
S_{0}(f)(x)=\left(\sum_{(\varepsilon, j, k) \in \Lambda_{n}} 2^{j n}\left|f_{j, k}^{\varepsilon}\right|^{2} \chi_{j, k}(x)\right)^{1 / 2} .
$$

We have $\|f\|_{L^{p}} \simeq\left\|S_{0}(f)\right\|_{L^{p}}$.

(1) Because $f \in M_{r, p}^{t, \tau}$, by Lemma 27,

$$
\left|f_{j_{u, s}, k}^{0}\right| \lesssim\left(1+j_{u, s}\right)^{-\tau} 2^{(r-n / 2) j_{u, s}} .
$$


By Lemma 29, we have $\left|g_{j_{u, s}, k}^{0}\right| \lesssim 2^{u-(r+t+n / 2) j_{u, s}}$. Hence we can get

$$
\begin{aligned}
& S_{0}\left(T_{1}\right)(x) \\
& =\left(\sum_{\left|Q_{j, k}\right|=\left|Q_{j_{u, s}, k}\right|} 2^{2 j_{u, s} n}\left|f_{j_{u, s}, k}^{0}\right|^{2}\left|g_{j_{u, s}, k}^{0}\right|^{2} \chi\left(2^{j_{u, s}} x-k\right)\right)^{1 / 2} \\
& \lesssim\left(\sum_{\left|Q_{j, k}\right|=\left|Q_{j_{u, s}, k}\right|} 2^{2 j_{u, s} n}\left(1+j_{u, s}\right)^{-2 \tau} 2^{2(r-n / 2) j_{u, s}}\right. \\
& \left.\times 2^{2 u-2(r+t+n / 2) j_{u, s}} 2^{2 j_{u, s}(n / 2+t)} \chi\left(2^{j_{u, s}} x-k\right)\right)^{1 / 2} .
\end{aligned}
$$

Because $j_{u, s} \geq 0$, we have

$$
\begin{gathered}
S_{0}\left(T_{1}\right)(x) \lesssim\left(1+j_{u, s}\right)^{-\tau} 2^{u}\left(\sum_{\left|Q_{j, k}\right|=\mid Q_{j, s}, k} \chi_{j, k}(x)\right)^{1 / 2}, \\
\left\|T_{1}\right\|_{L^{p}(\mathrm{Q})}=\left\|S_{0}\left(T_{1}\right)\right\|_{L^{p}(\mathrm{Q})} \lesssim\left(1+j_{u, s}\right)^{-\tau} 2^{u}|Q|^{1 / p} .
\end{gathered}
$$

(2) Now we estimate

$$
T_{2}(x)=\sum_{(\varepsilon, j, k) \in \Lambda_{n}, j \geq j_{u, s}|l| \leq \sqrt{n} 2^{(M+2) n}} f_{j, k+l}^{0} g_{j, k}^{\varepsilon} \Phi_{j, k+l}^{0}(x) \Phi_{j, k}^{\varepsilon}(x) .
$$

Because $j \geq j_{u, s} \geq 0$, we have

$$
\left|f_{j, k}^{0}\right| \lesssim\left(1+j_{\mathcal{u}, s}\right)^{-\tau} 2^{(r-n / 2) j} \leq\left(1+j_{u, s}\right)^{-\tau} 2^{(r+t-n / 2) j} .
$$

Let

$$
\begin{gathered}
\Lambda_{n, 2}^{Q}=\left\{(\varepsilon, j, k) \in \Lambda_{n}\left|j \geq j_{u, s}, \forall\right| l \mid \leq \sqrt{n} 2^{(M+2) n},\right. \\
\left.\left|\operatorname{supp}\left(\Phi_{j, k+l}^{0}(x) \Phi_{j, k}^{\varepsilon}(x)\right) \cap Q\right| \neq 0\right\} .
\end{gathered}
$$

Then, we can get

$$
\begin{aligned}
& S_{0}\left(T_{2}\right)(x)=\left(\sum_{(\varepsilon, j, k) \in \Lambda_{n, 2}^{Q}}\left|f_{j, k+l}^{0}\right|^{2}\left|g_{j, k}^{\varepsilon}\right|^{2} \chi_{j, k}(x)\right)^{1 / 2} \\
& \lesssim\left(\sum_{(\varepsilon, j, k) \in \Lambda_{n, 2}^{Q}} 2^{2 j n}(1+j)^{-2 \tau}\right. \\
&\left.\times 2^{2 j(r+t-n / 2)}\left|g_{j, k}^{\varepsilon}\right|^{2} \chi_{j, k}(x)\right)^{1 / 2} \\
& \\
& \lesssim\left(1+j_{u, s}\right)^{-\tau} S_{t+r}\left(g_{u}\right)(x) .
\end{aligned}
$$

Because $g_{u}(x)$ is a $\left(t+r, 2^{u}, E_{u}\right)$-combination atom,

$$
\begin{aligned}
\left\|S_{0}\left(T_{2}\right)\right\|_{L^{p}(Q)} & \lesssim\left(1+j_{u, s}\right)^{-\tau}\left\|S_{t+r}\left(g_{u}\right)\right\|_{L^{p}(Q)} \\
& \lesssim\left(1+j_{u, s}\right)^{-\tau} 2^{u}|Q|^{1 / p}
\end{aligned}
$$

(3) Since $g_{u}(x)$ is a $\left(t+r, 2^{u}, E_{u}\right)$-combination atom, for $s \geq 1,\left(i, m^{\prime}\right) \in H_{u, s}, j \geq 0$, and $Q=Q_{u, s, i, m^{\prime}}$,

$$
\left|g_{j, k}^{0}\right| \lesssim 2^{u-n j / 2} 2^{-(r+t) j}=2^{u-n j / 2}|Q|^{(r+t) / n} .
$$

Let

$$
\begin{gathered}
\Lambda_{n, 3}^{Q}=\left\{(\varepsilon, j, k) \in \Lambda_{n}\left|j \geq j_{u, s}, \forall\right| l \mid \leq \sqrt{n} 2^{(M+2) n},\right. \\
\left.\left|\operatorname{supp}\left(\Phi_{j, k}^{0} \Phi_{j, k+l}^{\varepsilon}\right) \cap Q\right| \neq 0\right\} .
\end{gathered}
$$

We have, by $j_{u, s} \geq 0$,

$$
\begin{aligned}
S_{0}\left(T_{3}\right)(x) & =\left(\sum_{(\varepsilon, j, k) \in \Lambda_{n, 3}^{Q}} 2^{j n}\left|g_{j, k}^{0}\right|^{2}\left|f_{j, k}^{\varepsilon}\right|^{2} \chi_{j, k}(X)\right)^{1 / 2} \\
& \lesssim 2^{u}|Q|^{(r+t) / n}\left(\sum_{(\varepsilon, j, k) \in \Lambda_{n, 3}^{Q}} 2^{j(n+2 t)}\left|f_{j, k}^{\varepsilon}\right|^{2} \chi_{j, k}(x)\right)^{1 / 2} .
\end{aligned}
$$

By the fact that $j \geq j_{u, s}$ and $f \in M_{r, p}^{t, \tau}\left(\mathbb{R}^{n}\right)$, we get

$$
\begin{aligned}
\left\|S_{0}\left(T_{3}\right)\right\|_{L^{p}(Q)} & \lesssim 2^{u}|Q|^{(r+t) / n}\left(1-\log _{2}|Q|\right)^{-\tau}|Q|^{1 / p-(r+t) / n} \\
& \lesssim 2^{u}\left(1+j_{u, s}\right)^{-\tau}|Q|^{1 / p} .
\end{aligned}
$$

(4) Now we estimate the term $T_{4}(x)$. Let

$$
\begin{gathered}
\Lambda_{n, 4}^{\mathrm{Q}}=\left\{(\varepsilon, j, k) \in \Lambda_{n} \mid j \geq j_{u, s}, \forall\left(\varepsilon, \varepsilon^{\prime}, j, k, l\right) \in \Lambda_{n}^{\prime},\right. \\
\left.\left|\operatorname{supp}\left(\Phi_{j, k+l}^{\varepsilon^{\prime}}(x) \Phi_{j, k}^{\varepsilon}(x)\right) \cap Q\right| \neq 0\right\} .
\end{gathered}
$$

Because $f \in M_{r, p}^{t, \tau}\left(\mathbb{R}^{n}\right)$, we have

$$
\begin{aligned}
\left|f_{j, k}^{\varepsilon}\right| \lesssim(1+j)^{-\tau} 2^{j(r-n / 2)} \lesssim(1+j)^{-\tau} 2^{j(r+t-n / 2)} & \\
S_{0}\left(T_{4}\right)(x) & =\left(\sum_{(\varepsilon, j, k) \in \Lambda_{n, 4}^{Q}} 2^{j n}(1+j)^{-2 \tau}\right. \\
& \left.\times 2^{j(2 r+2 t-n)} 2^{j n}\left|g_{j, k}^{\varepsilon}\right|^{2} \chi_{j, k}(x)\right)^{1 / 2} \\
& \leqslant\left(1+j_{u, s}\right)^{-\tau} S_{t+r}\left(g_{u}\right)(x) .
\end{aligned}
$$


Then we can get, by the fact that $g_{u}$ is a $\left(t+r, 2^{u}, E_{u}\right)$-combination atom,

$$
\begin{aligned}
& \left\|S_{0}\left(T_{4}\right)\right\|_{L^{p}(Q)} \\
& \quad \leq\left(1+j_{u, s}\right)^{-\tau}\left\|S_{t+r}\left(g_{u}\right)\right\|_{L^{p}(Q)} \leqslant 2^{u}\left(1+j_{u, s}\right)^{-\tau}|Q|^{1 / p} .
\end{aligned}
$$

(5) Now we estimate the term

$$
T_{5}(x)=\sum_{(\varepsilon, j, k) \in \Lambda_{n}, j \geq j_{u, s}} f_{j, k}^{\varepsilon} g_{j, k}^{\varepsilon}\left(\left(\Phi_{j, k}^{\varepsilon}(x)\right)^{2}-2^{n j / 2} \Phi_{j, k}^{0}(x)\right) .
$$

Because the function $\left(\Phi_{j, k}^{\varepsilon}\right)^{2}-2^{n j / 2} \Phi_{j, k}^{0}$ plays the role as that of $\Phi_{j, k}^{\varepsilon}(x)$, we have

$$
\left\|S_{0}\left(T_{5}\right)\right\|_{L^{p}(Q)} \leqslant 2^{u}\left(1+j_{u, s}\right)^{-\tau}|Q|^{1 / p} .
$$

(6) To estimate the term $T_{6}(x)$, we take $h \in L^{p^{\prime}}(Q)$. Let

$$
\begin{aligned}
\Lambda_{n, 6}^{\mathrm{Q}}=\left\{(\varepsilon, j, k) \in \Lambda_{n} \mid j \geq j_{u, s},\right. & \\
& \left.\left|\operatorname{supp}\left(\Phi_{j, k+l}^{0}(x) \Phi_{j, k}^{\varepsilon}(x)\right) \cap Q\right| \neq 0\right\} .
\end{aligned}
$$

By the orthogonality of the wavelet function, we have

$$
\begin{aligned}
\left\langle T_{6}, h\right\rangle & =\left\langle\sum_{(\varepsilon, j, k) \in \Lambda_{n, 6}^{Q}} f_{j, k}^{\varepsilon} g_{j, k}^{\varepsilon} 2^{j n / 2} \Phi_{j, k}^{0}, h\right\rangle \\
& =\sum_{(\varepsilon, j, k) \in \Lambda_{n, 6}^{Q}} f_{j, k}^{\varepsilon} g_{j, k}^{\varepsilon} 2^{n j / 2} h_{j, k}^{0} .
\end{aligned}
$$

Then,

$$
\begin{aligned}
\left|\left\langle T_{6}, h\right\rangle\right| & \leqslant \int \sum_{(\varepsilon, j, k) \in \Lambda_{n, 6}^{Q}} 2^{n j}\left|f_{j, k}^{\varepsilon} \| g_{j, k}^{\varepsilon}\right| 2^{n j / 2}\left|h_{j, k}^{0}\right| \chi_{j, k}(x) d x \\
& \lesssim \int \sum_{(\varepsilon, j, k) \in \Lambda_{n, 6}^{Q}} 2^{n j}\left|f_{j, k}^{\varepsilon} \| g_{j, k}^{\varepsilon}\right| \chi_{j, k}(x) M(h)(x) d x .
\end{aligned}
$$

By Hölder's inequality and $j \geq j_{u, s} \geq 0$, it can be deduced that

$$
\begin{aligned}
& \sum_{(\varepsilon, j, k) \in \Lambda_{n, 6}^{Q}} 2^{n j}\left|f_{j, k}^{\varepsilon}\right|\left|g_{j, k}^{\varepsilon}\right| \chi_{j, k}(x) \\
& \leq\left(\sum_{(\varepsilon, j, k) \in \Lambda_{n, 6}^{Q}} 2^{(n-2 t-2 r) j}\left|f_{j, k}^{\varepsilon}\right|^{2} \chi_{j, k}(x)\right)^{1 / 2} \\
& \quad \times\left(\sum_{(\varepsilon, j, k) \in \Lambda_{n, 6}^{Q}} 2^{(n+2 t+2 r) j}\left|g_{j, k}^{\varepsilon}\right|^{2} \chi_{j, k}(x)\right)^{1 / 2} \\
& \leqslant 2^{u}|Q|^{(r+t) / n}\left(\sum_{(\varepsilon, j, k) \in \Lambda_{n, 6}^{Q}} 2^{(n-2 t-2 r) j}\left|f_{j, k}^{\varepsilon}\right|^{2} \chi_{j, k}(x)\right)^{1 / 2} .
\end{aligned}
$$

Finally, we can get

$$
\begin{aligned}
& \left|\left\langle T_{6}, h\right\rangle\right| \\
& \lesssim 2^{u}|Q|^{(r+t) / n} \|\left(\sum_{(\varepsilon, j, k) \in \Lambda_{n, 6}^{Q}} 2^{(n-2 t-2 r) j}\right. \\
& \left.\times\left|f_{j, k}^{\varepsilon}\right|^{2} \chi_{j, k}(x)\right)^{1 / 2}\left\|_{L^{p}(\mathrm{Q})}\right\| h \|_{L^{p^{\prime}}(\mathrm{Q})} \\
& \lesssim 2^{u}|Q|^{(r+t) / n}\left|1-\log _{2}\right| Q \|^{-\tau}|Q|^{1 / p-(r+t) / n} \\
& \leqslant 2^{u}|Q|^{1 / p}\left(1+j_{u, s}\right)^{-\tau} \text {. }
\end{aligned}
$$

Because $h$ takes over all functions in $L^{p^{\prime}}(Q)$, it is obvious that

$$
\left\|S_{0}\left(T_{6}\right)\right\|_{L^{p}(Q)} \lesssim\left(1+j_{u, s}\right)^{-\tau} 2^{u}|Q|^{1 / p} .
$$

Step 2. Assume that $0 \leq t<1$. Let $g(x)=$ $\sum_{(\varepsilon, j, k) \in \Lambda_{n}} g_{j, k}^{\varepsilon} \Phi_{j, k}^{\varepsilon}(x)$ and

$$
S_{t}(g)(x)=\left(\sum_{(\varepsilon, j, k) \in \Lambda_{n}} 2^{j(2 t+n)}\left|g_{j, k}^{\varepsilon}\right|^{2} \chi_{j, k}(x)\right)^{1 / 2} .
$$

We need to prove that

$$
\left\|S_{t}\left(f g_{u}\right)\right\|_{L^{p}(Q)} \leqslant 2^{u}\left(1+j_{u, s}\right)^{-\tau}|Q|^{1 / p} .
$$

The index sets $\Lambda_{n, i}^{Q}, i=1,2, \ldots, 6$, are the same as Step 1 . (1) For the term $T_{1}$, we have

$$
\begin{aligned}
S_{t} & \left(T_{1}\right)(x) \\
& =\left(\sum_{\left|Q_{j, k}\right|=|=| Q_{j_{u, s}, k} \mid} 2^{j_{u, s}(n+2 t)} 2^{j_{u, s} n}\right.
\end{aligned}
$$

$$
\left.\times\left|f_{j_{u, s}, k}^{0}\right|^{2}\left|g_{j_{u, s}, k}^{0}\right|^{2} \chi\left(2^{j_{u, s}} x-k\right)\right)^{1 / 2}
$$

$\lesssim\left(\sum_{\left|Q_{j, k}\right|=\left|Q_{j_{u, s},}\right|} 2^{j_{u, s}(n+2 t)} 2^{j_{u, s} n}\left(1+j_{u, s}\right)^{-2 \tau} 2^{2(r-n / 2) j_{u, s}}\right.$

$$
\left.\times 2^{2 u-2(r+t+n / 2) j_{u, s}} 2^{2 j_{u, s}(n / 2+t)} \chi\left(2^{j_{u, s}} x-k\right)\right)^{1 / 2}
$$

$\lesssim\left(1+j_{u, s}\right)^{-\tau} 2^{u}\left(\sum_{\left|Q_{j, k}\right|=\left|Q_{j u, s, k}\right|} \chi_{j, k}(x)\right)^{1 / 2}$. 
Then, we have

$$
\left\|T_{1}\right\|_{H^{t, p}(\mathrm{Q})}=\left\|S_{t}\left(T_{1}\right)\right\|_{L^{p}(\mathrm{Q})} \leqslant\left(1+j_{u, s}\right)^{-\tau} 2^{u}|Q|^{1 / p} .
$$

(2) For the term

$$
T_{2}(x)=\sum_{(\varepsilon, j, k) \in \Lambda_{n}, j \geq j_{u, s}|l| \leq \sqrt{n} 2^{(M+2) n}} f_{j, k+l}^{0} g_{j, k}^{\varepsilon} \Phi_{j, k+l}^{0}(x) \Phi_{j, k}^{\varepsilon}(x),
$$

we have

$$
\begin{aligned}
& S_{t}\left(T_{2}\right)(x) \\
& =\left(\sum_{(\varepsilon, j, k) \in \Lambda_{n, 2}^{Q}} 2^{j(n+2 t)} 2^{j n}\left|f_{j, k+l}^{0}\right|^{2}\left|g_{j, k}^{\varepsilon}\right|^{2} \chi_{j, k}(x)\right)^{1 / 2} \\
& \leq\left(\sum_{(\varepsilon, j, k) \in \Lambda_{n, 2}^{Q}} 2^{j(n+2 t)} 2^{j n}(1+j)^{-2 \tau}\right. \\
& \left.\quad \times 2^{2 j(r-n / 2)}\left|g_{j, k}^{\varepsilon}\right|^{2} \chi_{j, k}(x)\right)^{1 / 2} \\
& \leq\left(1+j_{u, s}\right)^{-\tau} S_{t+r}\left(g_{u}\right)(x) .
\end{aligned}
$$

Because $g_{u}(x)$ is a $\left(t+r, 2^{u}, E_{u}\right)$-combination atom, we have

$$
\begin{aligned}
\left\|S_{t}\left(T_{2}\right)\right\|_{L^{p}(\mathrm{Q})} & \leq\left(1+j_{u, s}\right)^{-\tau}\left\|S_{t+r}\left(g_{u}\right)\right\|_{L^{p}(\mathrm{Q})} \\
& \leq\left(1+j_{u, s}\right)^{-\tau} 2^{u}|Q|^{1 / p} .
\end{aligned}
$$

(3) Because $g_{u}(x)$ is a $\left(t+r, 2^{u}, E_{u}\right)$-combination atom, for $s \geq 1,\left(i, m^{\prime}\right) \in H_{u, s}$, and $Q=Q_{u, s, i, m^{\prime}}$, we can get

$$
\begin{gathered}
\left|g_{j, k}^{0}\right| \leqslant 2^{u-n j / 2} 2^{-(r+t) j}=2^{u-n j / 2}|Q|^{(r+t) / n}, \\
S_{t}\left(T_{3}\right)(x) \\
\leqslant 2^{u}\left(\sum_{(\varepsilon, j, k) \in \Lambda_{n, 3}^{Q}} 2^{j(n+2 t)} 2^{-n j} 2^{-(r+t) j}\left|f_{j, k}^{\varepsilon}\right|^{2} \chi_{j, k}(x)\right)^{1 / 2} \\
\leqslant 2^{u}|Q|^{(r+t) / n}\left(\sum_{(\varepsilon, j, k) \in \Lambda_{n, 3}^{Q}} 2^{j(n+2 t)}\left|f_{j, k}^{\varepsilon}\right|^{2} \chi_{j, k}(x)\right)^{1 / 2} .
\end{gathered}
$$

From the fact that $j \geq j_{u, s}$ and $f \in M_{r, p}^{t, \tau}\left(\mathbb{R}^{n}\right)$, we deduce that

$$
\begin{aligned}
& \left\|S_{t}\left(T_{3}\right)\right\|_{L^{p}(Q)} \\
& \quad \leq 2^{u}|Q|^{(r+t) / n}\left(-\log _{2}|Q|\right)^{-\tau}|Q|^{1 / p-(r+t) / n} \leqslant 2^{u} j_{u, s}^{-\tau}|Q|^{1 / p} .
\end{aligned}
$$

(4) For the term $T_{4}(x)$, because $f \in M_{r, p}^{t, \tau}\left(\mathbb{R}^{n}\right)$ and $g_{u}$ is a $\left(t+r, 2^{u}, E_{u}\right)$-combination atom, we have $\left|f_{j, k}^{\mathcal{E}}\right| \lesssim(1+$ $j)^{-\tau} 2^{j(r-n / 2)}$ and

$$
\begin{aligned}
& S_{t}\left(T_{4}\right)(x)=\left(\sum_{(\varepsilon, j, k) \in \Lambda_{n, 4}^{Q}} 2^{j(n+2 t)}(1+j)^{-2 \tau}\right. \\
& \left.\times 2^{j(2 r-n)} 2^{j n}\left|g_{j, k}^{\varepsilon}\right|^{2} \chi_{j, k}(x)\right)^{1 / 2} \\
& \leqslant\left(1+j_{u, s}\right)^{-\tau} S_{t+r}\left(g_{u}\right)(x) .
\end{aligned}
$$

Then, we can get

$$
\begin{aligned}
& \left\|S_{t}\left(T_{4}\right)\right\|_{L^{p}(\mathrm{Q})} \\
& \quad \leqslant\left(1+j_{u, s}\right)^{-\tau}\left\|S_{t+r}\left(g_{u}\right)\right\|_{L^{p}(\mathrm{Q})} \leqslant 2^{u}\left(1+j_{u, s}\right)^{-\tau}|Q|^{1 / p} .
\end{aligned}
$$

(5) Now we estimate the term

$$
T_{5}(x)=\sum_{(\varepsilon, j, k) \in \Lambda_{n}, j \geq j_{u, s}} f_{j, k}^{\varepsilon} g_{j, k}^{\varepsilon}\left(\left(\Phi_{j, k}^{\varepsilon}(x)\right)^{2}-2^{n j / 2} \Phi_{j, k}^{0}(x)\right) .
$$

Because the function $\left(\Phi_{j, k}^{\varepsilon}(x)\right)^{2}-2^{n j / 2} \Phi_{j, k}^{0}(x)$ plays the role as that of $\Phi_{j, k}^{\varepsilon}(x)$, we have

$$
\left\|S_{t}\left(T_{5}\right)\right\|_{L^{p}(\mathrm{Q})} \leqslant 2^{u}\left(1+j_{u, s}\right)^{-\tau}|Q|^{1 / p} .
$$

(6) For the term $T_{6}(x)$, we take $h \in H^{-t, p^{\prime}}(Q)$. By the orthogonality of the wavelet functions, we have

$$
\begin{aligned}
\left\langle T_{6}, h\right\rangle & =\left\langle\sum_{(\varepsilon, j, k) \in \Lambda_{n, 6}^{Q}} f_{j, k}^{\varepsilon} g_{j, k}^{\varepsilon} 2^{j n / 2} \Phi_{j, k}^{0}, h\right\rangle \\
& =\sum_{(\varepsilon, j, k) \in \Lambda_{n, 6}^{Q}} f_{j, k}^{\varepsilon} g_{j, k}^{\varepsilon} 2^{n j / 2} h_{j, k}^{0} .
\end{aligned}
$$

We can get

$$
\begin{aligned}
\left|\left\langle T_{6}, h\right\rangle\right| & \leqslant\left|\int \sum_{(\varepsilon, j, k) \in \Lambda_{n}^{Q}} 2^{n j / 2} g_{j, k}^{\varepsilon} f_{j, k}^{\varepsilon} 2^{j n} h_{j, k}^{0} \chi_{j, k}(x) d x\right| \\
& \leqslant \int \sum_{(\varepsilon, j, k) \in \Lambda_{n}^{Q}} 2^{n j}\left|g_{j, k}^{\varepsilon}\right|\left|f_{j, k}^{\varepsilon}\right| M(h)(x) \chi_{j, k}(x) d x .
\end{aligned}
$$


By Hölder's inequality, we have

$$
\begin{aligned}
& \sum_{(\varepsilon, j, k) \in \Lambda_{n, 6}^{Q}} 2^{n j}\left|f_{j, k}^{\varepsilon}\right|\left|g_{j, k}^{\varepsilon}\right| \chi_{j, k}(x) \\
& \lesssim\left(\sum_{(\varepsilon, j, k) \in \Lambda_{n, 6}^{Q}} 2^{(n-2 t-2 r) j}\left|f_{j, k}^{\varepsilon}\right|^{2} \chi_{j, k}(x)\right)^{1 / 2} \\
& \quad \times\left(\sum_{(\varepsilon, j, k) \in \Lambda_{n, 6}^{Q}} 2^{(n+2 t+2 r) j}\left|g_{j, k}^{\varepsilon}\right|^{2} \chi_{j, k}(x)\right)^{1 / 2} \\
& \leqslant S_{t+r}\left(g_{u}\right)(x)\left(\sum_{(\varepsilon, j, k) \in \Lambda_{n, 6}^{Q}} 2^{(n-2 t-2 r) j}\left|f_{j, k}^{\varepsilon}\right|^{2} \chi_{j, k}(x)\right)^{1 / 2} .
\end{aligned}
$$

Because $j \geq j_{u, s}$ implies that $2^{-j(r+t)} \leq 2^{-j_{u, s}(r+t)}$, we can get

$$
\begin{aligned}
& \left|\left\langle T_{6}, h\right\rangle\right| \\
& \leq 2^{u}\left\|\left(\sum_{(\varepsilon, j, k) \in \Lambda_{n, 6}^{Q}} 2^{(n-2 t-2 r) j}\left|f_{j, k}^{\varepsilon}\right|^{2} \chi_{j, k}(x)\right)^{1 / 2}\right\|_{L^{p}(Q)} \\
& \quad \times\|h\|_{L^{p^{\prime}}(Q)} \\
& \leq\left. 2^{u}|Q|^{(r+t) / n}\left|1-\log _{2}\right| Q\right|^{-\tau}|Q|^{1 / p-(r+t) / n} \\
& \leq 2^{u}|Q|^{1 / p}\left(1+j_{u, s}\right)^{-\tau} .
\end{aligned}
$$

Because $h$ takes over all functions in $H^{-t, p^{\prime}}(Q)$, we can get

$$
\left\|S_{t}\left(T_{6}\right)\right\|_{L^{p}(Q)} \lesssim\left(1+j_{u, s}\right)^{-\tau} 2^{u}|Q|^{1 / p} .
$$

This completes the proof for the case $0 \leq t<1$.

Step 3. Now we consider the case $t \geq 1$. In this case, there exists an integer $[t]$ such that $[t] \leq t<[t]+1$. For any $\alpha=$ $\left(\alpha_{1}, \alpha_{2}, \ldots, \alpha_{n}\right) \in \mathbb{N}^{n}$ with $|\alpha|=\sum_{i=1}^{n} \alpha_{i}=[t]$, the derivative $\partial^{\alpha} / \partial x^{\alpha}$ of the product $f g_{u}$ can be represented as

$$
\frac{\partial^{\alpha}}{\partial x^{\alpha}}\left(f g_{u}\right)=\sum_{\gamma, \beta} C_{\alpha, \beta, \gamma}\left(\frac{\partial^{\beta}}{\partial x^{\beta}}\right) f(x) \frac{\partial^{\gamma}}{\partial x^{\gamma}} g_{u}(x),
$$

where $|\alpha|=|\beta|+|\gamma|$. Denote $\left(\partial^{\beta} / \partial x^{\beta}\right) f(x)$ and $\left(\partial^{\gamma} / \partial x^{\gamma}\right) g_{u}(x)$ by $f_{\beta}(x)$ and $g_{u, \gamma}(x)$, respectively. Applying the conclusion in Step 1, we only need to prove

$$
\left\|f_{\beta} g_{u, \gamma}\right\|_{H^{t-[t], p}} \leqslant\left(1+j_{u, s}\right)^{-\tau} 2^{u}|Q|^{1 / p},
$$

that is, $\left\|S_{t-[t]}\left(f_{\beta} g_{u, \gamma}\right)\right\|_{L^{p}} \lesssim 2^{u}\left(1+j_{u, s}\right)^{-\tau}|Q|^{1 / p}$.
If $g_{u}$ is a $\left(t+r, 2^{u}, E_{u}\right)$-combination atom, then

$$
\begin{aligned}
g_{u, \gamma}(x) & =\sum_{Q_{j, k} \in \mathscr{F}_{v, l}} g_{j, k}^{\varepsilon}\left(\frac{\partial}{\partial x}\right)^{\gamma}\left[2^{n j / 2} \Phi_{j, k}^{\varepsilon}(x)\right] \\
& =\sum_{Q_{j, k} \in \mathscr{F}_{v, l}} g_{j, k}^{\varepsilon} 2^{n j / 2} 2^{j|\gamma|}\left(\frac{\partial^{\gamma}}{\partial x^{\gamma}} \Phi^{\varepsilon}\right)\left(2^{j} x-k\right) .
\end{aligned}
$$

Hence,

$$
\begin{aligned}
S_{t-|\gamma|}\left(g_{u, \gamma}\right)(x) & =\left(\sum_{(\varepsilon, j, k) \in \Lambda_{n}} 2^{j(n+2 t-2|\gamma|)}\left|g_{j, k}^{\varepsilon}\right|^{2} 2^{j|\gamma|} \chi_{j, k}(x)\right)^{1 / 2} \\
& =\left(\sum_{(\varepsilon, j, k) \in \Lambda_{n}} 2^{j(n+2 t)}\left|g_{j, k}^{\varepsilon}\right|^{2} \chi_{j, k}(x)\right)^{1 / 2} \\
& =S_{t}\left(g_{u}\right)(x) .
\end{aligned}
$$

On the other hand, if $f \in M_{r, p}^{t, \tau}\left(\mathbb{R}^{n}\right)$, then

$$
\begin{aligned}
& \int_{Q}\left(\sum_{\varepsilon \in E_{n}, Q_{j, k} \subset Q} 2^{j(n+2 t)}\left|f_{j, k}^{\varepsilon}\right|^{2} \chi_{j, k}(x)\right)^{p / 2} d x \\
& \lesssim\left(1-\log _{2}|Q|\right)^{-p \tau}|Q|^{1-p(r+t) / n}
\end{aligned}
$$

and $\left(f_{\beta}\right)_{j, k}^{\varepsilon}=2^{j|\beta|} f_{j, k}^{\varepsilon}$. We have

$$
\begin{aligned}
& \int_{Q}\left(\sum_{\varepsilon \in E_{n}, Q_{j, k} \subset Q} 2^{j(n+2 t-2|\beta|)}\left|f_{j, k}^{\beta, \varepsilon}\right|^{2} \chi_{j, k}(x)\right)^{p / 2} d x \\
& \quad=\int_{Q}\left(\sum_{\varepsilon \in E_{n}, Q_{j, k} \subset Q} 2^{j(n+2 t-2|\beta|)} 2^{2 j|\beta|}\left|f_{j, k}^{\varepsilon}\right|^{2} \chi_{j, k}(x)\right)^{p / 2} d x \\
& \quad \lesssim\left(1-\log _{2}|Q|\right)^{-p \tau}|Q|^{1-p(t-|\beta|+r+|\beta|) / n}
\end{aligned}
$$

that is, $f_{\beta}(x) \in M_{r+|\beta|, p}^{t-|\beta|, \tau}\left(\mathbb{R}^{n}\right)$.

For any cube $Q$, the function $f_{\beta}(x)=$ $\sum_{(\varepsilon, j, k) \in \Lambda_{n}} f_{j, k}^{\beta, \varepsilon} \Phi_{j, k}^{\varepsilon}(x)$, and any dyadic cube $Q \subset S_{u}$, we divide the product $f_{\beta} \cdot g_{u, \gamma}$ into the following parts:

$$
\begin{aligned}
& T_{1}^{\beta, \gamma}(x)=\sum_{(\varepsilon, j, k) \in \Lambda_{n}, j=j_{u, s}|l| \leq \sqrt{n} 2^{(M+2) n}} f_{j, k+l}^{\beta, 0} g_{j, k}^{\gamma, 0} \Phi_{j, k+l}^{0}(x) \Phi_{j, k}^{0}(x), \\
& T_{2}^{\beta, \gamma}(x)=\sum_{(\varepsilon, j, k) \in \Lambda_{n}, j \geq j_{u, s}} \sum_{l l \mid \leq \sqrt{n} 2^{(M+2) n}} f_{j, k+l}^{\beta, 0} g_{j, k}^{\gamma, \varepsilon} \Phi_{j, k+l}^{0}(x) \Phi_{j, k}^{\varepsilon}(x), \\
& T_{3}^{\beta, \gamma}(x)=\sum_{(\varepsilon, j, k) \in \Lambda_{n}, j \geq j_{u, s}|l| \leq \sqrt{n}^{(M+2) n}} f_{j, k+l}^{\beta, \varepsilon} g_{j, k}^{\gamma, 0} \Phi_{j, k+l}^{\varepsilon}(x) \Phi_{j, k}^{0}(x),
\end{aligned}
$$




$$
\begin{gathered}
T_{4}^{\beta, \gamma}(x) \\
=\sum_{\left(\varepsilon, \varepsilon^{\prime}, j, k, l\right) \in \Lambda_{n}^{\prime}, j \geq j_{u, s}} \sum_{l l \mid \leq \sqrt{n} 2^{(M+2) n}} f_{j, k+l}^{\beta, \varepsilon^{\prime}} g_{j, k}^{\gamma, \varepsilon} \Phi_{j, k+l}^{\varepsilon^{\prime}}(x) \Phi_{j, k}^{\varepsilon}(x), \\
T_{5}^{\beta, \gamma}(x)=\sum_{(\varepsilon, j, k) \in \Lambda_{n}, j \geq j_{u, s}} f_{j, k}^{\beta, \varepsilon} g_{j, k}^{\gamma, \varepsilon}\left(\left(\Phi_{j, k}^{\varepsilon}(x)\right)^{2}-2^{n j / 2} \Phi_{j, k}^{0}(x)\right), \\
T_{6}^{\beta, \gamma}(x)=\sum_{(\varepsilon, j, k) \in \Lambda_{n}, j \geq j_{u, s}} f_{j, k}^{\beta, \varepsilon} g_{j, k}^{\gamma, \varepsilon} 2^{n j / 2} \Phi_{j, k}^{0}(x) .
\end{gathered}
$$

Similar to the method used in the case $0 \leq t<1$, we can complete the proof of the case $t \geq 1$. This completes the proof of this theorem.

By Theorem 30, we can get the following lemma.

Lemma 31. Given $r>0, t \geq 0,1<p<n /(r+t)$, and $\tau>$ $1 / p^{\prime}$, if $f \in M_{r, p}^{t, \tau}\left(\mathbb{R}^{n}\right)$ and $g_{u}$ is a $\left(t+r, 2^{u}, E_{u}\right)$-combination atom, then

$$
\left\|f g_{u}\right\|_{H^{t, p}} \lesssim(1+u)^{-\tau} 2^{u}\left|E_{u}\right|^{1 / p} .
$$

Theorem 32. Given $r>0, t \geq 0,1<p<n /(r+t)$, and $\tau>1 / p^{\prime}$, then $M_{r, p}^{t, \tau}\left(\mathbb{R}^{n}\right) \subset X_{r, p}^{t}\left(\mathbb{R}^{n}\right)$.

Proof. By Lemma 31, we have

$$
\begin{aligned}
\|f g\|_{H^{t, p}} & \lesssim \sum_{u \geq 0}(1+u)^{-\tau} 2^{u}\left|E_{u}\right|^{1 / p} \\
& \lesssim\left(\sum_{u \geq 0}(1+u)^{-p^{\prime} \tau}\right)^{1 / p^{\prime}}\left(\sum_{u \geq 0} 2^{p u}\left|E_{u}\right|\right)^{1 / p} \\
& \leq\|g\|_{H^{t+r, p} .}
\end{aligned}
$$

4.2. The Sharpness for $M_{r, p}^{t, \tau}\left(\mathbb{R}^{n}\right)$. In this section, applying our wavelet characterization of multiplier spaces and fractal theory, we prove that the scope of the index of $M_{r, p}^{t, \tau}\left(\mathbb{R}^{n}\right)$ obtained in Theorem 32 is sharp for $r+t<1$. Precisely, by Meyer wavelets, we construct a counterexample to show that Theorem 32 is not true for the case $0 \leq \tau \leq 1 / p^{\prime}$.

Our key idea is to construct a group of sets $C_{s}$ composed by special dyadic cubes and fractal set $H$ with Hausdorff dimension $n-(t+r) p$. Denote by $S_{s}$ the union $\bigcup_{Q \in C_{s}} Q$ and $H=\bigcap_{s \geq 1} S_{s}$. By the above dyadic cubes $S_{s}, s \geq 0$, we construct a special $L^{p}$ function $g(x)$, which is bounded on $S_{s} \backslash S_{s+1}$ for all $s \geq 1$. The fractional integration $I_{r+t} g(x)$ bumps on the fractal set $H$. Then we construct a multiplier $f(x)$ such that its wavelet coefficients are based on these special dyadic cubes $C_{s}$ for all $s \geq 1$. Applying our wavelet characterization of multiplier spaces, we prove that the product of the above multiplier $f(x)$ and the function $I_{r+t} g(x)$ will go out to the desired space $H^{t, p}\left(\mathbb{R}^{n}\right)$.

Theorem 33. If $0 \leq \tau \leq 1 / p^{\prime}$, there exists $f \in M_{r, p}^{t, \tau}\left(\mathbb{R}^{n}\right)$ such that $f \notin X_{r, p}^{t}\left(\mathbb{R}^{n}\right)$.
Proof. We use Meyer wavelets and suppose that $\varepsilon=(1,1$, $\ldots, 1)$ and $\Phi^{\varepsilon}(0)>0$. First of all, we construct a group of self-similar cubes $\left\{Q_{u}\right\}$ such that the limitation is a set with Hausdorff measure $n-(t+r) p$. that

We construct two series of integers $\left\{\tau_{s}\right\}_{s \geq 1}$ and $\left\{v_{s}\right\}_{s \geq 1}$ such

$$
\begin{gathered}
V_{1}=\max \left\{\left[\frac{4 n}{n-(t+r) p}\right], 2^{(M+2) n}\right\}, \\
1 \leq \tau_{s}<\left[\frac{4 n}{n-(t+r) p}\right] \\
\leq v_{s} \leq \max \left\{\left[\frac{5 n}{n-(t+r) p}\right], 2^{(M+2) n}\right\} .
\end{gathered}
$$

Denote $\sigma_{s}=\sum_{1 \leq i \leq s} \tau_{i}$ and $u_{s}=\sum_{1 \leq i \leq s} v_{i}$. We take $\tau_{s}$ such that there exists $C>0$ satisfying that

$$
\begin{aligned}
& 2^{n \sigma_{s}-(n-(t+r) p) u_{s}} \geq 2^{-n u_{1}}, \\
& \lim _{s \rightarrow+\infty} 2^{n \sigma_{s}-(n-(t+r) p) u_{s}}=C, \\
& n-(t+r) p=\lim _{s \rightarrow+\infty} \frac{n \sigma_{s}}{u_{s}} .
\end{aligned}
$$

For $\tau_{s}$ and $v_{s}$, denote $l_{s}=\left(l_{s, 1}, l_{s, 2}, \ldots, l_{s, n}\right) \in \mathbb{Z}_{s, 0}^{n}$ if $l_{s} \in \mathbb{Z}^{n}$, and for $i=1,2, \ldots, n$, we have

$$
0 \leq l_{s, i}<2^{\tau_{s}-1} \quad \text { or } 0 \leq l_{s, i}+2^{\tau_{s}-1}-2^{v_{s}}<2^{\tau_{s}-1} .
$$

Denote $\mathbb{Z}_{1}^{n}=\mathbb{Z}_{1,0}^{n}$, and, for $s \geq 2$, denote $k_{s} \in \mathbb{Z}_{s}^{n}$, if there exists $k_{s-1} \in \mathbb{Z}_{s-1}^{n}$ and $l_{s} \in \mathbb{Z}_{s, 0}^{n}$ such that $k_{s}=2^{v_{s}} k_{s-1}+l_{s}$.

We divide the unit dyadic cube $Q_{0}=[0,1]^{n}$ into $2^{n v_{1}}$ dyadic cubes. Then, we reserve the $2^{n \tau_{1}}$ dyadic cubes which are near the $2^{n}$ vertex points; that is, we reserve $C_{1}=\left\{Q_{v_{1}, l_{1}}\right.$ : $\left.l_{1} \in \mathbb{Z}_{1,0}^{n}\right\}$ and denote $x \in S_{1}$, if there exists $l_{1} \in \mathbb{Z}_{1,0}^{n}$ such that $x \in Q_{v_{1}, l_{1}}$.

For the dyadic cube $Q_{v_{1}, l_{1}} \in C_{1}$, we divide it into $2^{n v_{2}}$ dyadic cubes, and we reserve the $2^{n \tau_{2}}$ dyadic cubes which are near the $2^{n}$ vertex points; that is, we reserve

$$
C_{2, l_{1}}=\left\{Q_{u_{2}, 2^{v_{2}} l_{1}+l_{2}}, l_{2} \in \mathbb{Z}_{1,0}^{n}\right\} .
$$

For $s=2$, denote

$$
C_{2}=\left\{Q_{u_{2}, k_{2}}, k_{2}=2^{v_{2}} l_{1}+l_{2}, l_{1}, l_{2} \in \mathbb{Z}_{1,0}^{n}\right\}
$$

and denote $x \in S_{2}$ if there exists $k_{2} \in \mathbb{Z}_{2}^{n}$ such that $x \in Q_{u_{2}, k_{2}}$.

We continue this process until infinity, and we get a series of dyadic cubes $C_{s}$ and sets $S_{s}$. We know that $\left|S_{s}\right|=2^{n\left(\sigma_{s}-u_{s}\right)}$ and the limitation of $\left\{S_{s}\right\}$ is a fractal set $H$ with Hausdorff dimension $n-(t+r) p$.

For $s \geq 1$, let

$$
f_{s}(x)=\sum_{Q_{u_{s}, k_{s}} \subset C_{s}} f_{u_{s}, k_{s}}^{\varepsilon} \Phi_{u_{s}, k_{s}}^{\varepsilon}(x),
$$

where $f_{u_{s}, k_{s}}^{\varepsilon}=s^{-1 / p^{\prime}} 2^{-t u_{s}} 2^{(t+r) u_{s}}$. Let $f(x)=\sum_{s \geq 1} f_{s}(x)$. Applying the wavelet characterization of Morrey spaces, 
$f \in M_{r, p}^{t, 1 / p^{\prime}}\left(\mathbb{R}^{n}\right)$. In fact, for $s, l \geq 1$ and any cube $Q$ with $2^{-n u_{s+1}} \leq|Q|<2^{-n u_{s}}$, we have

$$
\left|Q \cap\left(S_{s+l} \backslash S_{s+l+1}\right)\right| \lesssim 2^{-n\left(\sigma_{s+l}-\sigma_{s}\right)-n u_{s}} .
$$

Hence, we get

$$
\begin{aligned}
& \int_{Q}\left(\sum_{Q_{u_{s}, k_{s}} \subset \mathrm{Q}} 2^{u_{s}(n+2 t)}\left|f_{u_{s}, k_{s}}^{\mathcal{E}}\right|^{2} \chi\left(2^{u_{s}} x-k_{s}\right)\right)^{p / 2} d x \\
& \lesssim \int_{Q \backslash S_{s+1}}\left(\sum_{Q_{u_{s}, k_{s}} \subset Q} 2^{u_{s}(n+2 t)} s^{-2 / p^{\prime}}\right. \\
& \left.\times 2^{-2 t u_{s}} 2^{2(r+t) u_{s}} \chi\left(2^{u_{s}} x-k_{s}\right)\right)^{p / 2} d x \\
& +\sum_{l \geq 1} \int_{Q \cap\left(S_{s+l} \backslash S_{S+l+1}\right)}\left(\sum_{Q_{u_{s}, k_{s}} \subset Q} 2^{u_{s}(n+2 t)} s^{-2 / p^{\prime}} 2^{-2 t u_{s}} 2^{2(r+t) u_{s}}\right. \\
& \left.\times \chi\left(2^{u_{s}} x-k_{s}\right)\right)^{p / 2} d x \\
& \lesssim s^{-p / p^{\prime}} 2^{(t+r) p u_{s}} 2^{-n u_{s}} \\
& +\sum_{l \geq 1}(s+l)^{-p / p^{\prime}} 2^{(t+r) p u_{s+l}} 2^{-n\left(\sigma_{s+l}-\sigma_{s}\right)-n u_{s}} \\
& \lesssim\left(1-\log _{2}|Q|\right)^{-p / p^{\prime}}|Q|^{1-(t+r) p / n} .
\end{aligned}
$$

Let $\delta$ be a sufficient small positive real number. For $x \in$ $[0,1]^{n} \backslash S_{1}$, then $g(x)=1$. For $s \geq 1$ and $x \in S_{s} \backslash S_{s+1}$, we take

$$
g(x)=s^{-1 / p}\left[\log _{2}(1+s)\right]^{-(1+\delta) / p} 2^{(t+r) u_{s}} .
$$

Then, we have $g \in L^{p}\left([0,1]^{n}\right)$. In fact,

$$
\begin{aligned}
& \int_{[0,1]^{n}}|g(x)|^{p} d x \\
& \quad=\int_{[0,1]^{n} \backslash S_{1}} g^{p}(x) d x+\sum_{s \geq 1} \int_{S_{s} \backslash S_{s+1}} g^{p}(x) d x \\
& \quad \lesssim 1+\sum_{s \geq 1} \int_{S_{s} \backslash S_{s+1}} s^{-1}\left[\log _{2}(1+s)\right]^{-(1+\delta)} 2^{(t+r) p u_{s}} d x \\
& \quad \lesssim \sum_{s \geq 1} s^{-1}\left[\log _{2}(1+s)\right]^{-(1+\delta)} 2^{(t+r) p u_{s}} 2^{n\left(\sigma_{s}-u_{s}\right)} \lesssim 1 .
\end{aligned}
$$

Now we estimate the coefficients $\left|g_{u_{s}, k_{s}}\right|$. We divide the estimate into two cases.
(1) For $\operatorname{dist}\left(Q_{u_{s}, k_{s}}, S_{s}\right) \leq 2^{-u_{s}}$,

$$
\begin{aligned}
g_{j, k}= & \int_{S_{s}} g(y)\left(2^{-u_{s}}+\left|y-2^{-u_{s}} k_{s}\right|\right)^{(t+r-n)} d y \\
& +\sum_{1 \leq l \leq s-1} \int_{S_{l} \backslash S_{l+1}} l^{-1 / p}\left[\log _{2}(1+l)\right]^{-(1+\delta) / p} 2^{n\left(u_{l}-\sigma_{l}\right) / p} \\
& \times\left(2^{-u_{l}}+\left|y-2^{-u_{l}} k_{l}\right|\right)^{(t+r-n)} d y \\
\gtrsim & s^{-1 / p}\left[\log _{2}(1+s)\right]^{-(1+\delta) / p} \\
& +\sum_{1 \leq l \leq s-1} s^{-1 / p}\left[\log _{2}(1+s)\right]^{-(1+\delta) / p} \\
\gtrsim & s^{1 / p^{\prime}}\left[\log _{2}(1+s)\right]^{-(1+\delta) / p} .
\end{aligned}
$$

(2) For $\operatorname{dist}\left(Q_{u_{s}, k_{s}}, S_{s}\right)>2^{-u_{s}}$, there exists $l<s$ such that $Q_{u_{s}, k_{s}} \subset S_{l-1} \backslash S_{l}$. It is easy to see that $g_{j, k}$ is equivalent to $l^{-1 / p^{\prime}}\left[\log _{2}(1+l)\right]^{-(1+\delta) / p}$

Finally, we have

$$
\begin{gathered}
\int\left(\sum_{(\varepsilon, j, k) \in \Lambda_{n}} 2^{j(n+2 t)}\left|g_{j, k}\right|^{2}\left|f_{j, k}^{\varepsilon}\right|^{2} \chi_{j, k}(x)\right)^{p / 2} d x \\
\gtrsim \sum_{s \geq 2}\left(\sum_{\operatorname{dist}\left(Q_{u_{s}, k_{s}}, S_{s}\right) \leq 2^{-u_{s}}} 2^{u_{s}(n+2 t)}\left|g_{u_{s}, k_{s}}\right|^{2}\right. \\
\left.\times\left|f_{u_{s}, k_{s}}^{\varepsilon}\right|^{2} \chi\left(2^{u_{s}} x-k_{s}\right)\right)^{p / 2} d x
\end{gathered}
$$

$$
\begin{aligned}
z \sum_{s \geq 2}( & \sum_{\operatorname{dist}\left(Q_{u_{s}, k_{s}}, S_{s}\right) \leq 2^{-u_{s}}} 2^{u_{s}(n+2 t)} s^{2 / p^{\prime}} \\
& \times\left[\log _{2}(1+s)\right]^{-2(1+\delta) / p} s^{-2 / p^{\prime}} \\
& \left.\times 2^{-2 t u_{s}+2(t+r) u_{s}} \chi\left(2^{u_{s}} x-k_{s}\right)\right)^{p / 2} d x
\end{aligned}
$$

$$
\begin{aligned}
& \gtrsim \sum_{s \geq 2}\left[\log _{2}(1+s)\right]^{-(1+\delta)} 2^{(t+r) p u_{s}} 2^{n\left(\sigma_{s}-u_{s}\right)} \\
& \gtrsim \sum_{s \geq 2}\left[\log _{2}(1+s)\right]^{-(1+\delta)}=\infty
\end{aligned}
$$

This completes the proof.

\section{An Application to Schrödinger Type Operators}

Let $L=I-\Delta+V$ be a Schrödinger operator. Maz'ya and Verbitsky [11] established many sufficient and necessary conditions such that $V$ is a multiplier from $H^{1,2}\left(\mathbb{R}^{n}\right)$ to $H^{-1,2}\left(\mathbb{R}^{n}\right)$. 
Their results can be used to study the Schrödinger operators L. In Section 4, Theorem 32 gives a relation between Morrey spaces and Sobolev multipliers. In this section, we give an application of the wavelet characterization of $X_{r, p}^{t}\left(\mathbb{R}^{n}\right)$ to the Schrödinger type operator $I+(-\Delta)^{r / 2}+V$.

For $V \in M_{r, p}^{t, \tau}\left(\mathbb{R}^{n}\right), \tau>1 / p^{\prime}$, and $g \in H^{t, p}\left(\mathbb{R}^{n}\right)$, we want to find a solution $f \in H^{t+r, p}\left(\mathbb{R}^{n}\right)$ to the equation

$$
\left(I+(-\Delta)^{r / 2}+V\right) f(x)=g(x) .
$$

Remark 34. Fixed $r>0, t \geq 0$, and $1<p<n /(r+t)$.

(i) If there exists a $\delta>0$ such that $\|V\|_{C^{r+t+\delta}}$ is sufficiently small, according to the continuity of CalderónZygmund operator $\left(I+(-\Delta)^{r / 2}\right)\left(I+(-\Delta)^{r / 2}+V\right)^{-1}$, (185) can be solved easily. But if we consider a nonsmooth potential $V \in M_{r, p}^{t, \tau}\left(\mathbb{R}^{n}\right)$, applying the same proof in Lemmas 8 and 9 , it is possible that $V$ is not a $L^{\infty}$ function.

(ii) The condition $\tau>1 / p^{\prime}$ cannot be weaken to $\tau \geq 1 / p^{\prime}$. In fact, according to our counterexample in Section 5 , if $r+t<1$, there exists some $V \in M_{r, p}^{t, 1 / p^{\prime}}\left(\mathbb{R}^{n}\right)$ such that the operator $I+(-\Delta)^{r / 2}+V$ is not continuous from $H^{t+r, p}\left(\mathbb{R}^{n}\right)$ to $H^{t, p}\left(\mathbb{R}^{n}\right)$.

Now, we use our sufficient condition of multiplier spaces $X_{r, p}^{t}\left(\mathbb{R}^{n}\right)$ to get the solution of (185). We need the following two operators. For $t, r>0$, let

$$
\begin{gathered}
T_{t, r}=\left[I+(-\Delta)^{t / 2}, I+(-\Delta)^{r / 2}\right], \\
S_{t, r}=\left[I+(-\Delta)^{t / 2}\right] V T_{t, r}^{-1} .
\end{gathered}
$$

Lemma 35. Given $r>0, t \geq 0,1<p<n /(r+t)$, and $\tau>1 / p^{\prime}$, if $V \in M_{r, p}^{t, \tau}\left(\mathbb{R}^{n}\right)$, the operator $S_{t, r}$ is bounded from $L^{p}\left(\mathbb{R}^{n}\right)$ to $L^{p}\left(\mathbb{R}^{n}\right)$ with the operator norm less than $C_{t, r, \tau, p}\|V\|_{M_{r, p}^{t, \tau}}$, where $C_{t, r, \tau, p}$ denotes a constant associated with $t, r, \tau$, and $p$.

Proof. By Theorem 32, for any $f \in L^{p}\left(\mathbb{R}^{n}\right)$, we have

$$
\begin{aligned}
\left\|S_{t, r} f\right\|_{L^{p}} & \lesssim\left\|\left[I+(-\Delta)^{t / 2}\right] V T_{t, r}^{-1} f\right\|_{L^{p}} \lesssim\left\|V T_{t, r}^{-1} f\right\|_{H^{t, p}} \\
& \lesssim C_{r, t, \tau, p}\|V\|_{M_{r, p}^{t, \tau}}^{t, \tau} T_{t, r}^{-1} f\left\|_{H^{t+r, p}} \lesssim C_{r, t, \tau, p}\right\| V\left\|_{M_{r, p}^{t, \tau}}\right\| f \|_{L^{p}} .
\end{aligned}
$$

Then, $S_{t, r}$ is a bounded operator on $L^{p}\left(\mathbb{R}^{n}\right)$ with the norm less than $C_{r, t, \tau, p}\|V\|_{M_{r, p}^{t, \tau}}$.

In the following lemma, we prove that $\left(I+S_{t, r}\right)$ is invertible in $L^{p}\left(\mathbb{R}^{n}\right)$ and the inverse $\left(I+S_{t, r}\right)^{-1}$ can be written formally as $\sum_{n=0}^{\infty}(-1)^{n} S_{t, r}^{n}$.

Lemma 36. Given $r>0, t \geq 0,1<p<n /(r+t)$, and $\tau>1 / p^{\prime}$, if $\|V\|_{M_{r, p}^{t, \tau}}<1 / C_{r, t, \tau, p}$, the operator $I+S_{t, r}$ is invertible in $L^{p}\left(\mathbb{R}^{n}\right)$.
Proof. By Lemma 35, $S_{t, r}$ is bounded on $L^{p}\left(\mathbb{R}^{n}\right)$. Hence, for any $f \in L^{p}\left(\mathbb{R}^{n}\right)$,

$$
\begin{aligned}
\left\|\sum_{n=0}^{\infty}(-1)^{n} S_{t, r}^{n} f\right\|_{L^{p}} & \lesssim \sum_{n=0}^{\infty}\left\|S_{t, r}^{n} f\right\|_{L^{p}} \\
& \lesssim \sum_{n=0}^{\infty}\left(C_{r, t, \tau, p}\|V\|_{M_{r, p}^{t, \tau}}\right)^{n}\|f\|_{L^{p}} .
\end{aligned}
$$

If $\|V\|_{M_{r, p}^{t, \tau}}<1 / C_{r, t, \tau, p}$, the above series is convergent in $L^{p}\left(\mathbb{R}^{n}\right)$. Further,

$$
\left(I+S_{t, r}\right)\left[\sum_{n=0}^{\infty}(-1)^{n} S_{t, r}^{n}\right]=\sum_{n=0}^{\infty}(-1)^{n} S_{t, r}^{n}-\sum_{n=1}^{\infty}(-1)^{n} S_{t, r}^{n}=I .
$$

Similarly, we can also get

$$
\left[\sum_{n=0}^{\infty}(-1)^{n} S_{t, r}^{n}\right]\left(I+S_{t, r}\right)=I
$$

that is, the operator $I+S_{t, r}$ is invertible in $L^{p}\left(\mathbb{R}^{n}\right)$.

Theorem 37. Given $r>0, t \geq 0,1<p<n /(r+t)$, and $\tau>1 / p^{\prime}$, if $\|V\|_{M_{r, p}^{t, \tau}}<1 / C_{r, t, \tau, p}$, then, for $g \in H^{t, p}\left(\mathbb{R}^{n}\right)$, there exists a unique solution $f \in H^{r+t, p}\left(\mathbb{R}^{n}\right)$ for (185).

Proof. Because $g \in H^{t, p}\left(\mathbb{R}^{n}\right)$, we have $\tilde{g}=\left(I+(-\Delta)^{t / 2}\right) g \in$ $L^{p}\left(\mathbb{R}^{n}\right)$. By Lemma 36, the operator $\left(I+S_{t, r}\right)$ is invertible in $L^{p}\left(\mathbb{R}^{n}\right)$. Hence, we can get that there exists a unique solution to the following equation in $L^{p}\left(\mathbb{R}^{n}\right)$ :

$$
\left(I+S_{t, r}\right) \widetilde{f}=\tilde{g}
$$

where $\widetilde{g} \in L^{p}\left(\mathbb{R}^{n}\right)$. Hence, for the above $\widetilde{g} \in L^{p}\left(\mathbb{R}^{n}\right)$,

$$
\tilde{f}=\left(I+S_{t, r}\right)^{-1} \tilde{g}=\left(I+S_{t, r}\right)^{-1}\left(I+(-\Delta)^{t / 2}\right) g(x) \in L^{p}\left(\mathbb{R}^{n}\right)
$$

is a solution to (191). Write $f=\left(I+(-\Delta)^{r / 2}\right)^{-1}\left(I+(-\Delta)^{t / 2}\right)^{-1} \widetilde{f}$. Then, $\tilde{f} \in L^{p}\left(\mathbb{R}^{n}\right)$ is equivalent to $f \in H^{r+t, p}\left(\mathbb{R}^{n}\right)$. It is easy to verify that $f$ is a solution to (185). This completes the proof.

\section{Conflict of Interests}

The authors declare that they have no conflict of interests in this submitted paper.

\section{Acknowledgments}

The research is supported by NSFC nos. 11171203, 11201280, and 11271209; New Teachers' Fund for Doctor Stations, Ministry of Education 20114402120003; Guangdong Natural Science Foundation S2011040004131; MYRG116(Y1-L3)-FST13QT; MYRG115(Y1-L4)-FST13-QT; and FDCT 098/2012/A3. 


\section{References}

[1] V. Maz'ya and T. O. Shaposhnikova, Theory of Multipliers in Spaces of Differentiable Functions, vol. 23 of Monographs and Studies in Mathematics, Pitman, 1985.

[2] Y. Liang, Y. Sawano, T. Ullrich, D. Yang, and W. Yuan, "New characterizations of Besov-Triebel-Lizorkin-Hausdorff spaces including coorbits and wavelets," The Journal of Fourier Analysis and Applications, vol. 18, no. 5, pp. 1067-1111, 2012.

[3] Y. Liang, Y. Sawano, T. Ullrich, D. Yang, and W. Yuan, "A new framework for generalized Besov-type and Triebel-Lizorkintype spaces," Dissertationes Mathematicae (Rozprawy Matematyczne), vol. 489, 114 pages, 2013.

[4] H. Triebel, Local Function Spaces, Heat and Naiver-Stokes Equations, vol. 20 of EMS Tracts in Mathematics, European Mathematical Society, Zürich, Switzerland, 2013.

[5] W. Yuan, W. Sickel, and D. Yang, "On the coincidence of certain approaches to smoothness spaces related to Morrey spaces," Mathematische Nachrichten, 2013.

[6] Q. Yang, "Characterization of multiplier spaces with Daubechies wavelets," Acta Mathematica Scientia B, vol. 32, no. 6, pp. 2315-2321, 2012.

[7] D. Yang and Y. Zhou, "Localized Hardy spaces $H^{1}$ related to admissible functions on $\mathrm{RD}$-spaces and applications to Schrödinger operators," Transactions of the American Mathematical Society, vol. 363, no. 3, pp. 1197-1239, 2011.

[8] C. L. Fefferman, "The uncertainty principle," Bulletin of the American Mathematical Society, vol. 9, no. 2, pp. 129-206, 1983.

[9] P. G. Lemarié-Rieusset, Recent Developments in the NavierStokes Problem, vol. 431, Chapman \& Hall, Boca Raton, Fla, USA, 2002.

[10] P. G. Lemarié-Rieusset, "Multipliers and Morrey spaces," Potential Analysis, vol. 38, no. 3, pp. 741-752, 2013.

[11] V. G. Maz'ya and I. E. Verbitsky, "The Schrödinger operator on the energy space: boundedness and compactness criteria," Acta Mathematica, vol. 188, no. 2, pp. 263-302, 2002.

[12] R. Jiang, D. Yang, and Y. Zhou, "Localized Hardy spaces associated with operators," Applicable Analysis, vol. 88, no. 9, pp. 14091427, 2009.

[13] V. G. Maz'ya and I. E. Verbitsky, "Capacitary inequalities for fractional integrals, with applications to partial differential equations and Sobolev multipliers," Arkiv för Matematik, vol. 33, no. 1, pp. 81-115, 1995.

[14] D. Yang and D. Yang, "Characterizations of localized $B M O\left(R^{n}\right)$ via commutators of localized Riesz transforms and fractional integrals associated to Schrödinger operators," Collectanea Mathematica, vol. 61, no. 1, pp. 65-79, 2010.

[15] D. Yang, D. Yang, and Y. Zhou, "Endpoint properties of localized Riesz transforms and fractional integrals associated to Schrödinger operators," Potential Analysis, vol. 30, no. 3, pp. 271-300, 2009.

[16] D. Yang, D. Yang, and Y. Zhou, "Localized BMO and BLO spaces on RD-spaces and applications to Schrödinger operators," Communications on Pure and Applied Analysis, vol. 9, no. 3, pp. 779-812, 2010.

[17] D. Yang, D. Yang, and Y. Zhou, "Localized Morrey-Campanato spaces on metric measure spaces and applications to Schrödinger operators," Nagoya Mathematical Journal, vol. 198, pp. 77-119, 2010.

[18] Y. Meyer, Ondelettes et Opérateurs, I et II, Hermann, Paris, France, 1992.
[19] P. Wojtaszczyk, A Mathematical Introduction to Wavelets, vol. 37 of London Mathematical Society Student Texts, Cambridge University Press, 1997.

[20] R. Coifman, P.-L. Lions, Y. Meyer, and S. Semmes, "Compensated compactness and Hardy spaces," Journal de Mathématiques Pures et Appliquées. Neuvième Série, vol. 72, no. 3, pp. 247-286, 1993.

[21] H. Triebel, Theory of Function Spaces, vol. 78 of Monographs in Mathematics, Birkhäuser, Basel, Switzerland, 1983.

[22] W. Yuan, W. Sickel, and D. Yang, Morrey and Campanato Meet Besov, Lizorkin and Triebel, vol. 2005 of Lecture Notes in Mathematics, Springer, Berlin, Germany, 2010.

[23] Q. Yang, Wavelet and Distribution, Beijing Science and Technology Press, 2002.

[24] Q. X. Yang, Z. X. Chen, and L. Z. Peng, "Uniform characterization of function spaces by wavelets," Acta Mathematica Scientia A, vol. 25, no. 1, pp. 130-144, 2005.

[25] D. Yang and W. Yuan, "A new class of function spaces connecting Triebel-Lizorkin spaces and Q spaces," Journal of Functional Analysis, vol. 255, no. 10, pp. 2760-2809, 2008.

[26] D. Yang and W. Yuan, "New Besov-type spaces and TriebelLizorkin-type spaces including Q spaces," Mathematische Zeitschrift, vol. 265, no. 2, pp. 451-480, 2010.

[27] J. Xiao, "Homothetic variant of fractional Sobolev space with application to Navier-Stokes system," Dynamics of Partial Differential Equations, vol. 4, no. 3, pp. 227-245, 2007.

[28] M. Essén, S. Janson, L. Peng, and J. Xiao, "Q spaces of several real variables," Indiana University Mathematics Journal, vol. 49, no. 2, pp. 575-615, 2000.

[29] G. Dafni and J. Xiao, "Some new tent spaces and duality theorems for fractional Carleson measures and $Q_{\alpha}\left(\mathbb{R}^{n}\right)$," Journal of Functional Analysis, vol. 208, no. 2, pp. 377-422, 2004.

[30] G. Dafni and J. Xiao, "The dyadic structure and atomic decomposition of $Q$ spaces in several real variables," The Tohoku Mathematical Journal, vol. 57, no. 1, pp. 119-145, 2005.

[31] L. Peng and Q. Yang, "Predual spaces for Q spaces," Acta Mathematica Scientia B, vol. 29, no. 2, pp. 243-250, 2009.

[32] Z. Wu and C. Xie, "Q spaces and Morrey spaces," Journal of Functional Analysis, vol. 201, no. 1, pp. 282-297, 2003.

[33] Y. Meyer and Q. X. Yang, "Continuity of Calderón-Zygmund operators on Besov or Triebel-Lizorkin spaces," Analysis and Applications, vol. 6, no. 1, pp. 51-81, 2008.

[34] Q. Yang, "Atomic decomposition in $L^{p}(1<p<\infty)$," Indian Journal of Pure and Applied Mathematics, vol. 31, no. 9, pp. 10811087, 2000. 


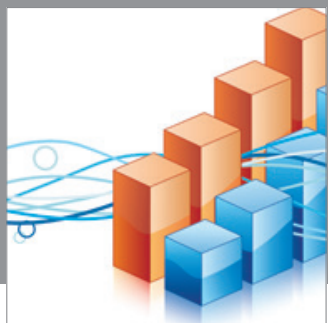

Advances in

Operations Research

mansans

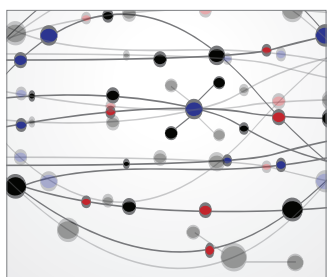

The Scientific World Journal
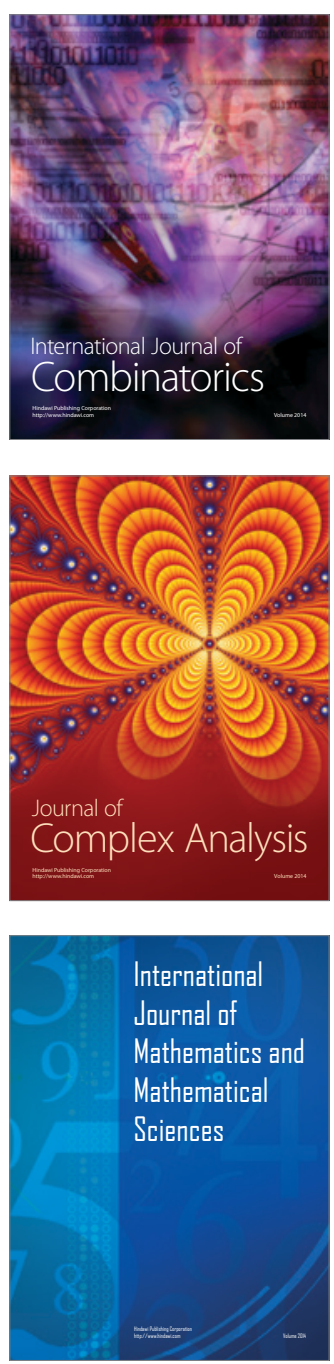
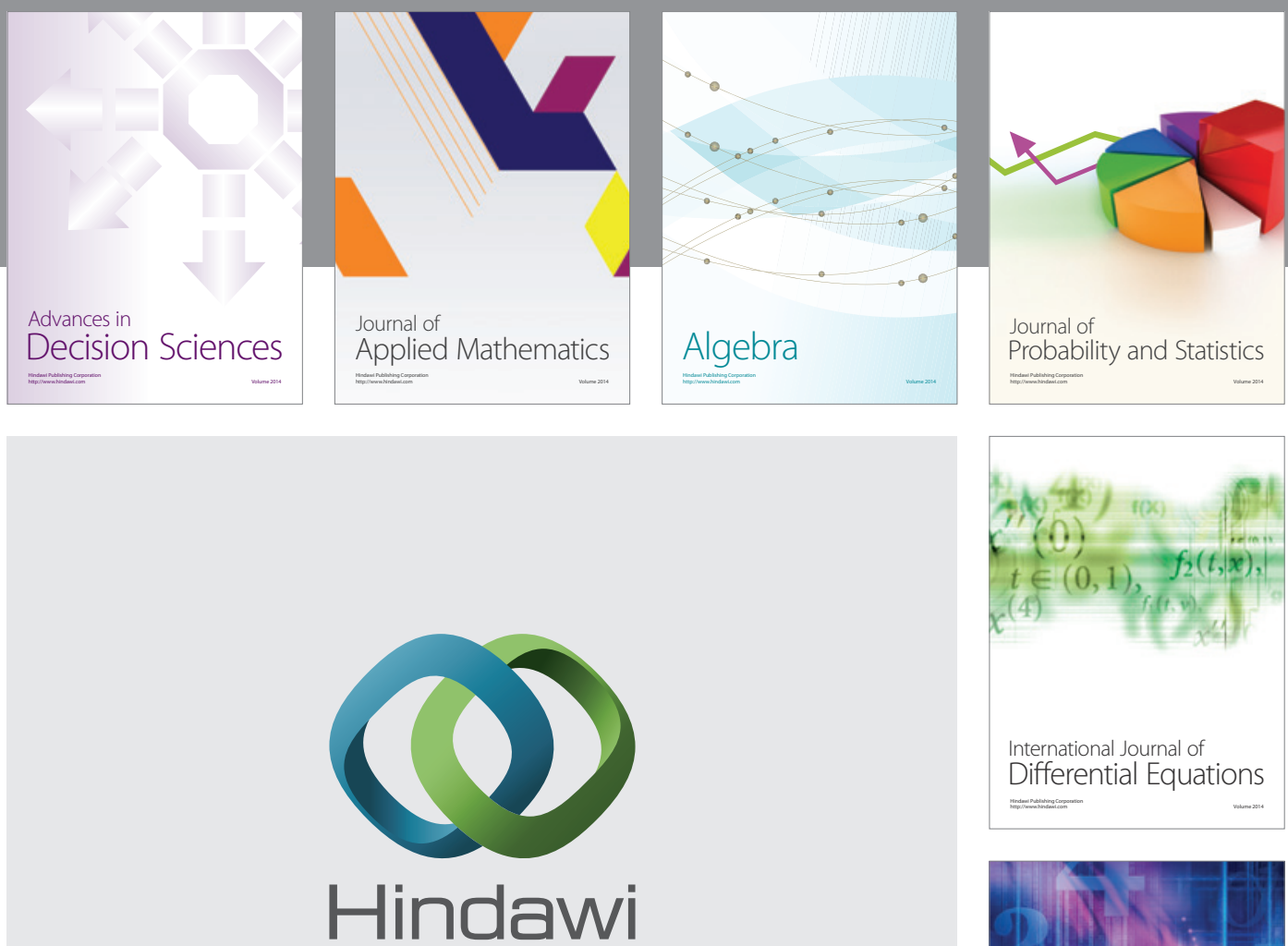

Submit your manuscripts at http://www.hindawi.com
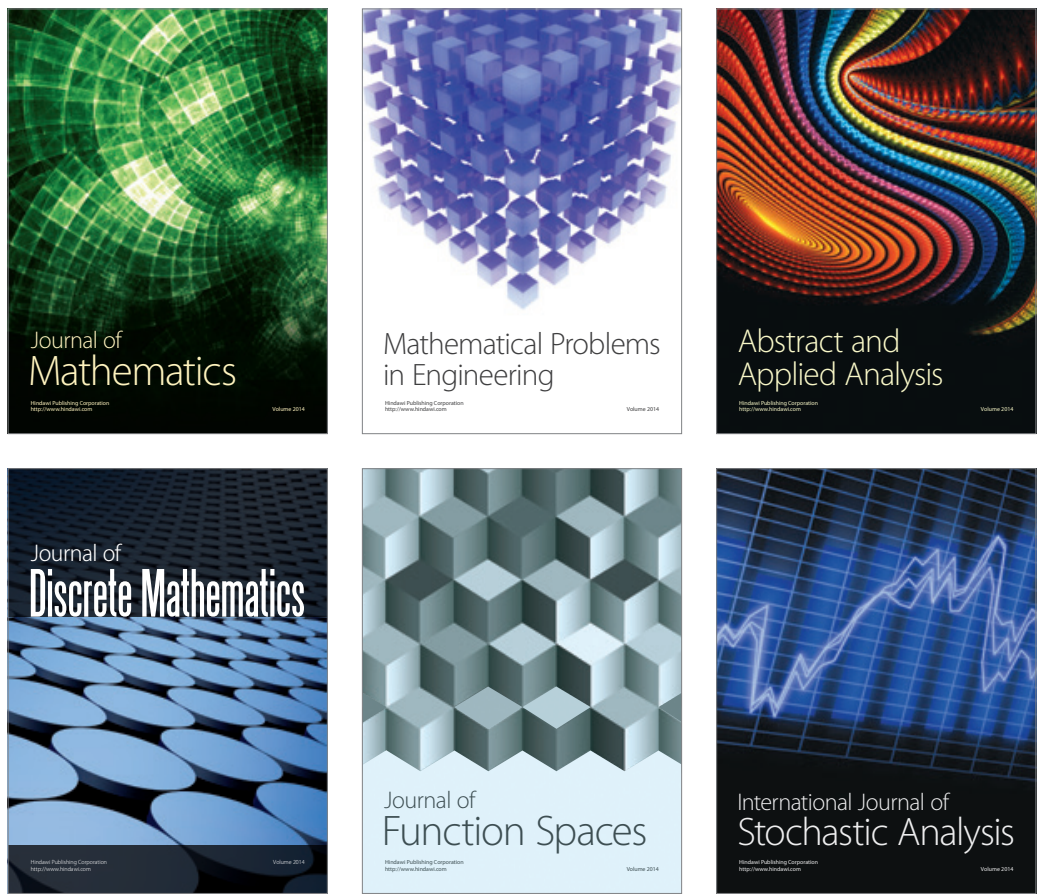

Journal of

Function Spaces

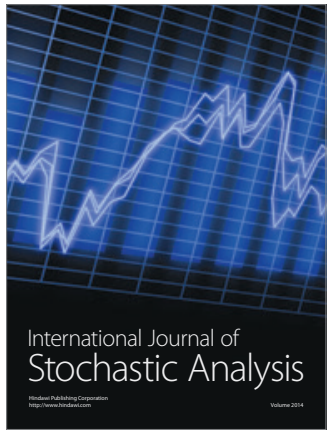

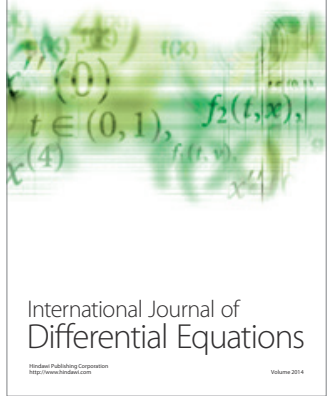
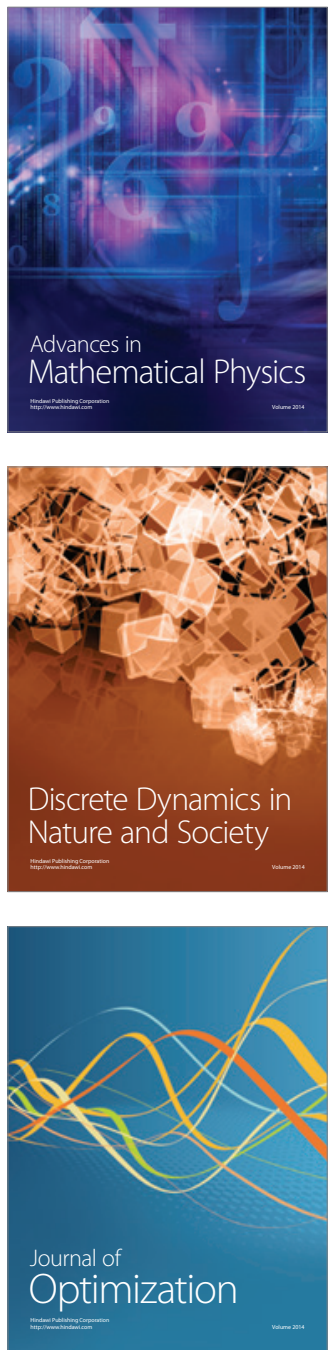